\title{
The mosquito Aedes aegypti has a large genome size and high transposable element load but contains a low proportion of transposon-specific piRNAs
}

Peter Arensburger ${ }^{1}$, Robert H Hice ${ }^{1}$, Jennifer A Wright ${ }^{1}$, Nancy L Craig ${ }^{2}$ and Peter W Atkinson ${ }^{1 *}$

\begin{abstract}
Background: The piRNA pathway has been shown in model organisms to be involved in silencing of transposons thereby providing genome stability. In D. melanogaster the majority of piRNAs map to these sequences. The medically important mosquito species Aedes aegypti has a large genome size, a high transposon load which includes Miniature Inverted repeat Transposable Elements (MITES) and an expansion of the piRNA biogenesis genes. Studies of transgenic lines of Ae. aegypti have indicated that introduced transposons are poorly remobilized and we sought to explore the basis of this. We wished to analyze the piRNA profile of Ae. aegypti and thereby determine if it is responsible for transposon silencing in this mosquito.

Results: Estimated piRNA sequence diversity was comparable between Ae. aegypti and D. melanogaster, but surprisingly only $19 \%$ of mosquito piRNAs mapped to transposons compared to $51 \%$ for D. melanogaster. Ae. aegypti piRNA clusters made up a larger percentage of the total genome than those of $D$. melanogaster but did not contain significantly higher percentages of transposon derived sequences than other regions of the genome. Ae. aegypti contains a number of protein coding genes that may be sources of piRNA biogenesis with two, traffic jam and maelstrom, implicated in this process in model organisms. Several genes of viral origin were also targeted by piRNAs. Examination of six mosquito libraries that had previously been transformed with transposon derived sequence revealed that new piRNA sequences had been generated to the transformed sequences, suggesting that they may have stimulated a transposon inactivation mechanism.

Conclusions: Ae. aegypti has a large piRNA complement that maps to transposons but primarily gene sequences, including many viral-derived sequences. This, together the more uniform distribution of piRNA clusters throughout its genome, suggest that some aspects of the piRNA system differ between Ae. aegypti and D. melanogaster.
\end{abstract}

\section{Background}

Research into the genome-wide regulation of transposons in model organisms such as Drosophila melanogaster, zebrafish and mice has revealed the importance of two small RNA pathways for controlling their movement thereby preserving genome stability [1-8]. This is especially important considering the abundance and diversity of transposons in eukaryote genomes in which

\footnotetext{
* Correspondence: peter.atkinson@ucr.edu

${ }^{1}$ Center for Disease Vector Research, Institute for Integrative Genome Biology, and Department of Entomology, University of California, Riverside, CA 92521, USA

Full list of author information is available at the end of the article
}

unregulated movement of active elements, or the nonautonomous sequences they can re-mobilize, would lead to insertion mutagenesis throughout the genome resulting in a decrease in genetic fitness. In D. melanogaster the Piwi-interacting RNAs (piRNAs) appear to function primarily in the regulation of transposons in both the germ line and in somatic tissues that envelope the ovaries although it is clear that transposons are not the only genomic targets of piRNAs [9-13]. In addition, endosiRNAs have been shown to target transposons in somatic tissues [10]. However, the D. melanogaster genome has a relatively small transposon load (only $3.86 \%$ of the $120 \mathrm{Mb}$ euchromatic DNA and $77 \%$ of $24 \mathrm{Mb}$ of

\section{Ciomed Central}


sequenced heterochromatic DNA making an average of $15.8 \%$ across $144 \mathrm{Mb}$ of the sequenced genome) $[14,15]$ compared with many other organisms. It is not clear how similar the transposon regulatory mechanisms between it and insects with much larger genome sizes and higher transposon loads might be.

The mosquito Aedes aegypti has a genome size of 1.38 Gb of which nearly half (47\%) is composed of transposons [16]. It is a vector of several human pathogens, most notably RNA viruses responsible for dengue and yellow fever, and so is an insect pest of high medical importance. Ae aegypti is somewhat amenable to modern genetic analysis through the use of transposonmediated genetic transformation, site-specific recombinases and RNAi leading to the emergence of novel control strategies based on the manipulation of its genome [17-20]. A curiosity is that, while genetic transformation of Ae. aegypti has been achieved using the exogenous transposons piggyBac, Mos1 and Hermes, none of these appear to re-mobilized at a frequency which allows the implementation of transposon-based genetic strategies such as gene tagging, and gene and enhancer trapping [21-24]. Indeed the failure of piggyBac to retain even somatic activity in transgenic lines of Ae. aegypti in which the piggyBac transposase is expressed is in contrast to the use of piggyBac as an incisive genetic tool in D. melanogaster, Triobolium castaneum and mice in which its mobility properties allow the identification of genes and regulatory sequences based on function [24-27]. Understanding the basis of the inactivity of these exogenous transposons in Ae. aegypti is important since the ability to use these (or other transposons) as mutagens is preventing the implementation of transposon-based genetic screens used to identify genes and sequences based on function. One possible explanation for the immobility of these three exogenous transposons once they have integrated into the Ae. aegypti genome is that they are rapidly silenced by the host's small RNA system.

Our knowledge of small RNA silencing of transposons in insects is based on studies from D. melanogaster. Two pathways, the piRNA and endo-siRNA pathway, are involved however many aspects of how these pathways actually function remain unknown. The endosiRNA pathway is dicer-dependent and generates $21 \mathrm{nt}$ small RNAs that target transposons in somatic and germ line tissues, including the follicle cells of the ovary $[10,12]$. They are also generated in Kc and S2 cells as well as the heads of adult flies and so are most likely dispersed through the somatic tissue of the insect [10].

The piRNA pathway generates small RNAs that are typically 24-30 nt in length and in Drosophila appears to be mainly devoted to the silencing of transposable elements $([1,2,6-8,28,29]$. In $D$. melanogaster it requires the action of three genes, Piwi, aubergine $(a u b)$ and Argonaute 3 (Ago3), the expression of the later two being confined to the germ line $[6,30]$. The piRNA pathway itself is proposed to consist of two pathways, the primary piRNA pathway and the amplification or pingpong pathway, the later acting in germ line tissues $[31,32]$. In $D$. melanogaster the primary piRNA pathway utilizes antisense transcripts generated from chromosomal clusters of piRNA target sequences or transposons that are then loaded onto the Piwi protein or, to a lesser extent the Aub protein, the later acting in the germ line. These piRNAs are 2'-O-methylated and act as guides to transcripts generated from either invading transposons or transposons (or genes) located in the clusters, thereby achieving suppression of transposition [33,34]. The pingpong pathway requires the action of the AGO3 and Aub proteins with sense transcripts derived from piRNA target sequences or transposons being loaded onto the AGO3 protein where their 3'ends are also 2'-O-methylated. Antisense transcripts originating from the same loci are loaded onto the Aub protein. Working in concert, both complexes are then capable of recognizing and slicing transcripts arising from target sequences and, at the same time, generating piRNAs that fuel subsequent amplification cycles. The outcome is an effective means by which transposons are silenced in the germ line thereby increasing genome stability. The presence of piRNA clusters in the genome also provides a memory of transposon invasions of the genome that is preserved in the female germ line and so can provide some level of immunity to the subsequent invasion of transposons recognized by the incumbent piRNA machinery $[9,35]$. However in D. melanogaster it is believed that this immunity takes more than one generation to develop before it affords resistance to at least some transposons [35]. Cytotype regulation of $P$ transposon transposition has been proposed to be controlled by, in part, the generation of piRNAs to the $P$ transposon $[35,36]$.

Interestingly the Ae. aegypti genome contains an expansion of the Piwi gene family with there being a single Ago3 gene and six Piwi genes [37]. Our analysis of Ae. aegypti Piwil indicates that it is a truncated gene and so may not be functional. It is not possible to exactly discern which Piwi corresponds to Drosophila $A u b$ although, based on sequence similarity, we believe Piwi2 is the most likely candidate. Ae. aegypti contains single dicer 2, ago 2 and dicer 1 genes but two ago 1 genes. By inference from studies in D. melanogaster these most likely play roles in the siRNA (dicer 2 and ago2) and miRNA (dicer 1 and ago1) pathways [38].

We wished to determine the piRNA complement of Ae. aegypti and to examine whether this small RNA regulatory pathway may be responsible for the control of 
transposons in this mosquito, whether piRNAs were generated from a few large clusters as they are in $D$. melanogaster, and whether piRNAs are also generated to protein coding genes. We report the results of sequencing seven Ae. aegypti small RNA libraries from five $A$ e. aegypti lines (including four transgenic lines) and one $D$. melanogaster library using high-throughput sequencing. We show that piRNA sequences are generated from piRNA clusters and from certain protein coding genes. Remarkably for an organism with such a high transposon load we show that a much lower percentage of Ae. aegypti piRNAs map to transposons than in $D$. melanogaster. Indeed the majority of piRNAs appear to be targeted to protein coding genes, some of which are of viral origin.

\section{Results}

\section{Library descriptions}

We sequenced seven Ae. aegypti libraries from five different mosquito lines (two lines were sequenced twice) as well as a single $D$. melanogaster library (Table 1 ). Four of these lines were transgenic and contained the Hermes, Mos1 or piggyBac transposase placed under the control of either the Ae. aegypti ß2-tubulin promoter or the D. melanogaster hsp70 promoter [23,39-41]. These four transgenic strains were designed and constructed for the separate purpose of determining whether these transposases could, using a jumpstarter strategy, remobilize their target transposons. All three experiments failed to detect significant levels of remobilization $[21,23,41]$ (Smith and Atkinson, unpublished). The Hermes and Mos1 expressing strains were constructed in 2007 and we estimated that they had each been maintained for approximately 35 generations under selection before RNA was obtained from them for small RNA library construction. Both the Hermes and Mos 1 tranposase strains were generated in the Orlando strain of $A e$. aegypti maintained at UC Riverside. The piggyBac line was generated in the Liverpool strain of Ae. aegypti, which is also the reference genomic strain [16]. The $D$. melanogaster strain was transgenic for the same autonomous Hermes transposon present in the autoHermes 257 Ae aegypti transgenic line and had been maintained at UC Riverside since 2002. The starting material for all libraries was whole tissue adults since we wished to determine the small RNA complement directed to transposons from both germ line and somatic tissues.

While there were some differences in total sequencing size between Ae. aegypti libraries, all produced similar size distribution patterns after removal of sequences matching ribosomal RNAs and miRNAs (Figures 1A, Additional file 1 Figure S1). Both Ae. aegypti and D. melanogaster libraries had sharp peaks at $21 \mathrm{nt}$ and broader peaks between 24 and $31 \mathrm{nt}$. The $21 \mathrm{nt}$ peaks matched previously described siRNA peaks in $D$. melanogaster $[9,42]$. The second peak was slightly shifted between the Ae. aegypti and D. melanogaster libraries. In D. melanogaster this peak was centered around 25 nt while in Ae. aegypti it was centered around $28 \mathrm{nt}$, the same distribution as seen for piRNAs in Bombyx mori and Danio rerio [5,43] (Additional file 1, Figure S1). The 25 nt centered peak in $D$. melanogaster matched the previously reported piRNA peak of this species [6]. The Ae. aegypti small RNAs between 24 - 31 nt exhibited bias for U at their 5' end and was observed for these small RNAs targeting transposons, gene and remaining sequences further supporting that these were likely to be piRNAs (Figure 1B).

Previously, $42 \%$ of D. melanogaster piRNAs from ovary libraries were reported to match transposon sequences and our whole adult tissue D. melanogaster library yielded a similar percentage (50\%)[6]. However only $19 \%$ of Ae. aegypti piRNAs matched known transposons despite these occupying some $47 \%$ of its genome (Table 2 and Figure 1C). In both D. melanogaster and Ae. aegypti the vast majority of transposon-matching piRNAs mapped inside gene boundaries (Figure 1A) with transposon-matching piRNAs in Ae. aegypti mapping predominantly to the anti-sense transcription strand $(72 \%$ of all piRNAs and $69 \%$ of uniquely mapping piRNAs). A similar pattern has been reported for $D$. melanogaster $[7,44]$.

Table 1 Ae. aegypti and D. melanogaster lines used in this study

\begin{tabular}{cccc}
\hline Library number & Species & Strain & Line (transformation plasmid) \\
\hline 1 & Ae. aegypti & Orlando & pMos3DB2Her \\
\hline 4 & Ae. aegypti & Orlando & pMos3DB2Her \\
\hline 2 & Ae. aegypti & Orlando & pBac3EB2Mos \\
\hline 6 & Ae. aegypti & Orlando & whoc3EB2Mos adults \\
\hline 10 & Ae. aegypti & Orlando type adults & whole adults \\
\hline 11 & Ae. aegypti & Orlando & whole adults \\
\hline 12 & Ae. aegypti & Liverpool & whole adults \\
\hline 14 & D. melanogaster & csW & whole adults Hermes
\end{tabular}




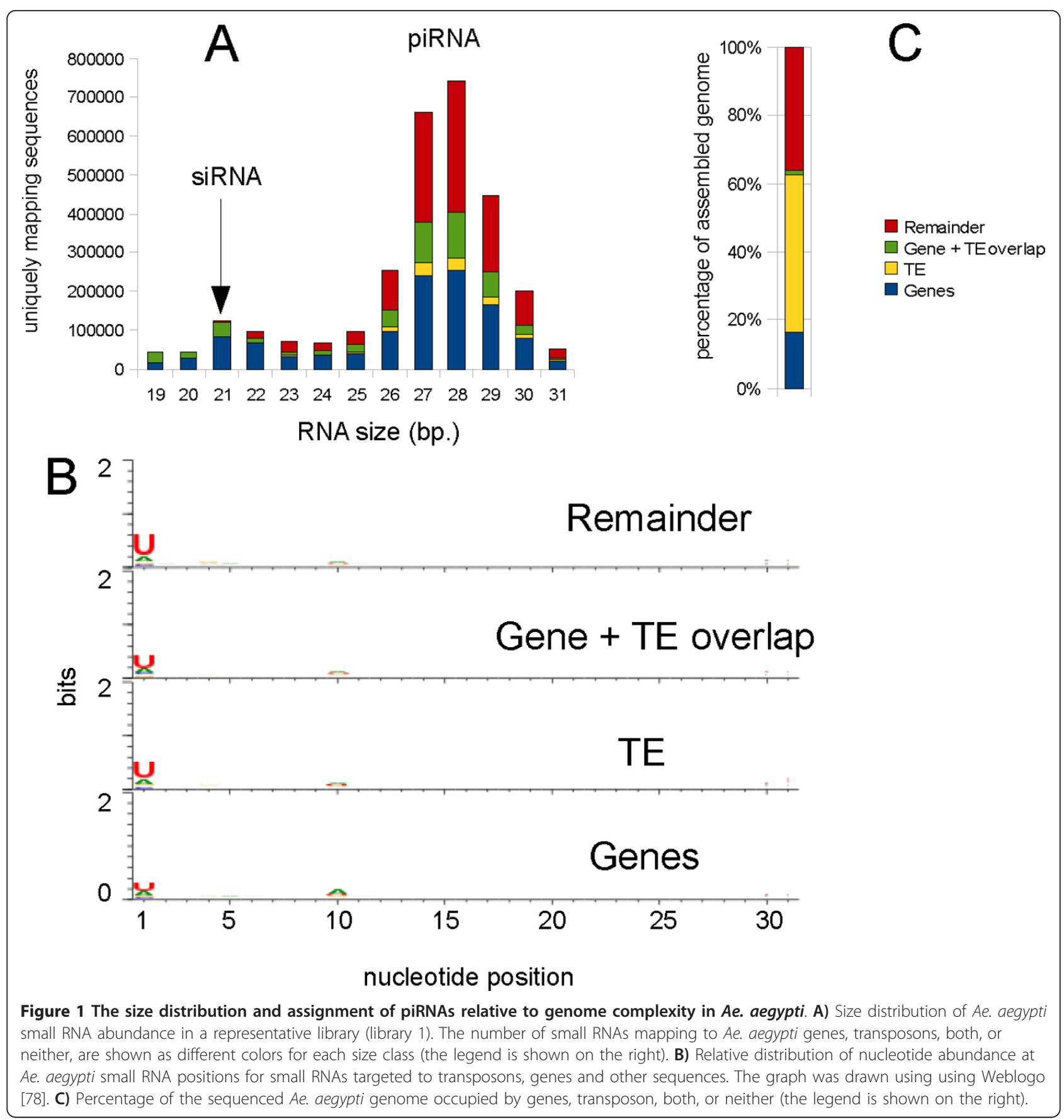

To determine if some of these small RNAs contained the $10 \mathrm{bp}$ overlap ping-pong signature seen for piRNAs specific to the $D$. melanogaster germ line we plotted the distance between the 5' ends of complementary small RNAs present in Ae. aegypti. We also performed the same analysis on small RNAs from our D. melanogaster library and found that $13.5 \%$ of these piRNAs contained this ping-pong signature, a number less than the $20 \%$ reported previously from libraries prepared from dissected ovaries, the difference most likely being due to ovaries being enriched for germ line specific piRNAs with this overlap [6]. In Ae. aegypti the same 10 bp overlap was also found in $19.5 \%$ of piRNAs that map to opposite strands and have at least one common nucleotide position, however the proportion of piRNAs with $U$ at the first position was higher in Ae. aegypti than seen in piRNAs obtained from our D. melanogaster library (Figure 2). These data suggest that a biochemical mechanism at least similar in function to the ping-pong amplification loop characterized in $D$. melanogaster also exists in Ae. aegypti however our data 
Table 2 Overlap of individual D. melanogaster piRNA clusters from Brennecke i. (2007) with one or more clusters from the present study

\begin{tabular}{|c|c|c|c|c|c|c|c|c|c|}
\hline \multicolumn{4}{|c|}{ Top piRNA clusters Brennecke et al. (2007) } & \multicolumn{6}{|c|}{ piRNA clusters from present study } \\
\hline Cluster id $^{1}$ & Chr. & start & stop & Cluster Unique piRNAs ${ }^{2}$ & Cluster id $^{1}$ & Chr. & start & stop & Cluster Unique piRNAs $^{2}$ \\
\hline \multirow[t]{2}{*}{1} & $2 R$ & 2144349 & 2386719 & 1686 & 1 & $2 R$ & 2110440 & 2217150 & 2350 \\
\hline & & & & & 2 & $2 \mathrm{R}$ & 2304581 & 2394124 & 1975 \\
\hline \multirow[t]{2}{*}{2} & $x$ & 21392175 & 21431907 & 986 & 24 & $x$ & 21390160 & 21406447 & 231 \\
\hline & & & & & 37 & $x$ & 21424709 & 21429775 & 194 \\
\hline 3 & 4 & 1258473 & 1348320 & 684 & 27 & 4 & 1250143 & 1289828 & 217 \\
\hline \multirow[t]{3}{*}{5} & $2 \mathrm{~L}$ & 20148259 & 20227581 & 482 & 22 & $2 \mathrm{~L}$ & 20136345 & 20153079 & 235 \\
\hline & & & & & 14 & $2 \mathrm{~L}$ & 20164387 & 20176739 & 333 \\
\hline & & & & & 12 & $2 \mathrm{~L}$ & 20198129 & 20230518 & 392 \\
\hline 6 & $3 \mathrm{~L}$ & 23273964 & 23314199 & 228 & 9 & $3 \mathrm{~L}$ & 23251131 & 23313773 & 520 \\
\hline 7 & U & 4015849 & 4029971 & 176 & 7 & $U$ & 4022887 & 4029272 & 834 \\
\hline \multirow[t]{4}{*}{8} & $x$ & 21505666 & 21684449 & 170 & 4 & $x$ & 21496075 & 21510974 & 1046 \\
\hline & & & & & 8 & $x$ & 21526569 & 21543427 & 520 \\
\hline & & & & & 33 & $x$ & 21631078 & 21672693 & 206 \\
\hline & & & & & 69 & $x$ & 21604048 & 21622362 & 123 \\
\hline 9 & $x$ & 21759393 & 21844063 & 155 & 53 & $x$ & 21786358 & 21878191 & 150 \\
\hline 10 & U & 5766708 & 5772171 & 133 & 1246 & $U$ & 5760625 & 5774759 & 6 \\
\hline 11 & $3 R$ & 27895169 & 27905030 & 107 & $\mathrm{~N} / \mathrm{A}^{3}$ & $3 R$ & $\mathrm{~N} / \mathrm{A}$ & $\mathrm{N} / \mathrm{A}$ & $\mathrm{N} / \mathrm{A}$ \\
\hline \multirow[t]{3}{*}{12} & 3LHet & 1402377 & 1557939 & 102 & 148 & 3LHet & 1438260 & 1446518 & 55 \\
\hline & & & & & 99 & 3LHet & 1485097 & 1535498 & 84 \\
\hline & & & & & 163 & 3LHet & 1557765 & 1579687 & 50 \\
\hline \multirow[t]{3}{*}{13} & 3LHet & 2011004 & 2180268 & 86 & 75 & 3LHet & 2068714 & 2118742 & 110 \\
\hline & & & & & 133 & 3LHet & 2126549 & 2158540 & 62 \\
\hline & & & & & 3480 & 3LHet & 2167493 & 2167493 & 1 \\
\hline 14 & U & 7542733 & 7545114 & 84 & 236 & U & 7532042 & 7545038 & 33 \\
\hline 15 & 3LHet & 238123 & 332969 & 71 & N/A & 3LHet & N/A & N/A & $\mathrm{N} / \mathrm{A}$ \\
\hline
\end{tabular}

${ }^{1}$ Clusters were ordered and numbered according to the abundance of cluster unique piRNAs in both studies. Cluster 6 from Brennecke et al. (2007) was not reported here because it was not located on an assembled portion of the published D. melanogaster BGDP 5 genome.

${ }^{2 " C l}$ Cluster unique piRNAs" were defined as piRNA sequences that mapped in only one location in the genome and inside a cluster.

${ }^{3}$ "N/A" indicates the absence of any overlapping piRNA cluster in present study.

do not enable us to comment on its tissue-specificity nor on the proteins specifically involved in its generation.

\section{piRNAs in Ae. aegypti are modified}

In both $D$. melanogaster and mice piRNAs the 3' terminal ribonucleotide contains a 2'-O-methyl modification that occurs after loading onto the Piwi proteins, a process catalyzed by the dmHEN1/Pimet protein [33,34]. Small RNAs containing this modification are resistant to periodate oxidation and $B$-elimination $[7,33]$. We purified small RNAs 28-31 nt in size and performed ß-elimination on them following periodate treatment and saw no change in their mobility, suggesting that their 3' ends were modified, consistent with them being piRNAs (Figure 3).

\section{Estimating piRNA abundance in Ae. aegypti}

To our knowledge a single estimate of total piRNA abundance in an organism has been published to date: it being estimated that the mouse genome contains a pool $2 \times 10^{5}$ potential piRNA sequences [45]. We attempted to perform similar estimates for the Ae aegypti libraries. In order to minimize the chances that sequencing artifacts might strongly affect our results we limited our estimates to piRNA sequences that matched the published Ae. aegypti genome along their entire length without mismatches. Using these criteria our Ae. aegypti libraries contained $1,563,634$ unique piRNA sequences. Furthermore, the majority of these sequences were only found in one of the five mosquito lines (Figure 4) suggesting that our sequencing efforts had sampled only a portion of a much larger piRNA pool. Betel et al. (2007) estimated the size of the mouse piRNA pool by extrapolating from the size of the sequenced libraries and the amount of overlap between library pairs. Applying a similar methodology (see Methods), we estimated the size of the Ae. aegypti piRNA pool to $1.7 \times 10^{7}$ (minimum estimate $5.5 \times 10^{6}$, maximum 

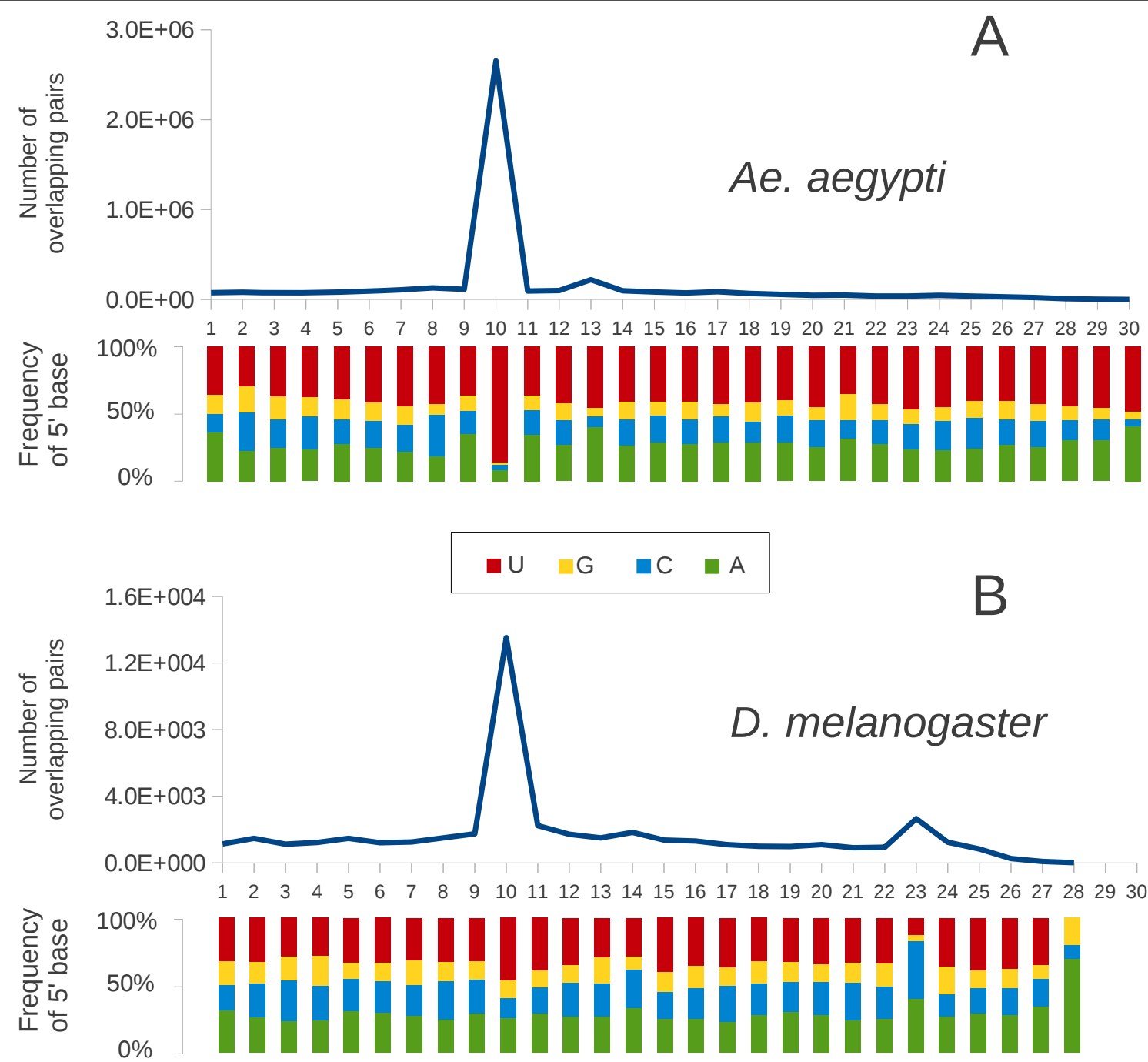

Figure 2 Size distribution and frequency of $5^{\prime}$ base of overlapping small RNAs (small RNAs at least 24 nt long, overlapping pairs on opposite strands) for all combined Ae. aegypti libraries (A) and the D. melanogaster library (B). The length of overlap is shown on the horizontal axes. Indicated above each axis is the number of possible overlapping pairs of small RNAs (individual small RNA sequences may be involved in multiple pairs) with specified overlap size. Indicated below each axis is the relative frequency of the $5^{\prime}$ base identity for sequences involved in overlapping pairs. The color code for bases is indicated in the center box.

estimate $2.3 \times 10^{7}$ ) potential piRNA sequences. We also estimated the size of the piRNA pool in D. melanogaster based on eight published small RNA libraries derived from Drosophila ovaries [46]. This yielded a similar estimate of a pool of $1.6 \times 10^{7}$ (minimum estimate $3.9 \times 10^{6}$, maximum estimate $2.2 \times 10^{7}$ ) piRNA sequences in $D$. melanogaster.

We conclude that the pool of different Ae. aegypti piRNA sequences was two orders of magnitude larger than in mice but found no evidence that it was different in size from D. melanogaster. We note the mouse and Ae. aegypti libraries were not derived from the same tissue types (the mouse libraries were derived from testes), but barring a very large difference between piRNAs in mouse testes and other mouse tissues, this should not fundamentally affect our conclusions.

\section{piRNA Clusters}

Validating cluster discovery methodology using the $D$. melanogaster library

piRNA clusters are believed to be the biogenesis sites of many piRNAs. D. melanogaster and $D$. virilis are to date, the only insects in which the location of piRNA clusters within their genomes has been published [9,31,47] (Brennecke 2007, Malone et al. 2009, Rozkov et al. 2010). piRNA clusters are typically identified by mapping the location of sequenced piRNA from ovary libraries to the genome assembly sequence. We sought 


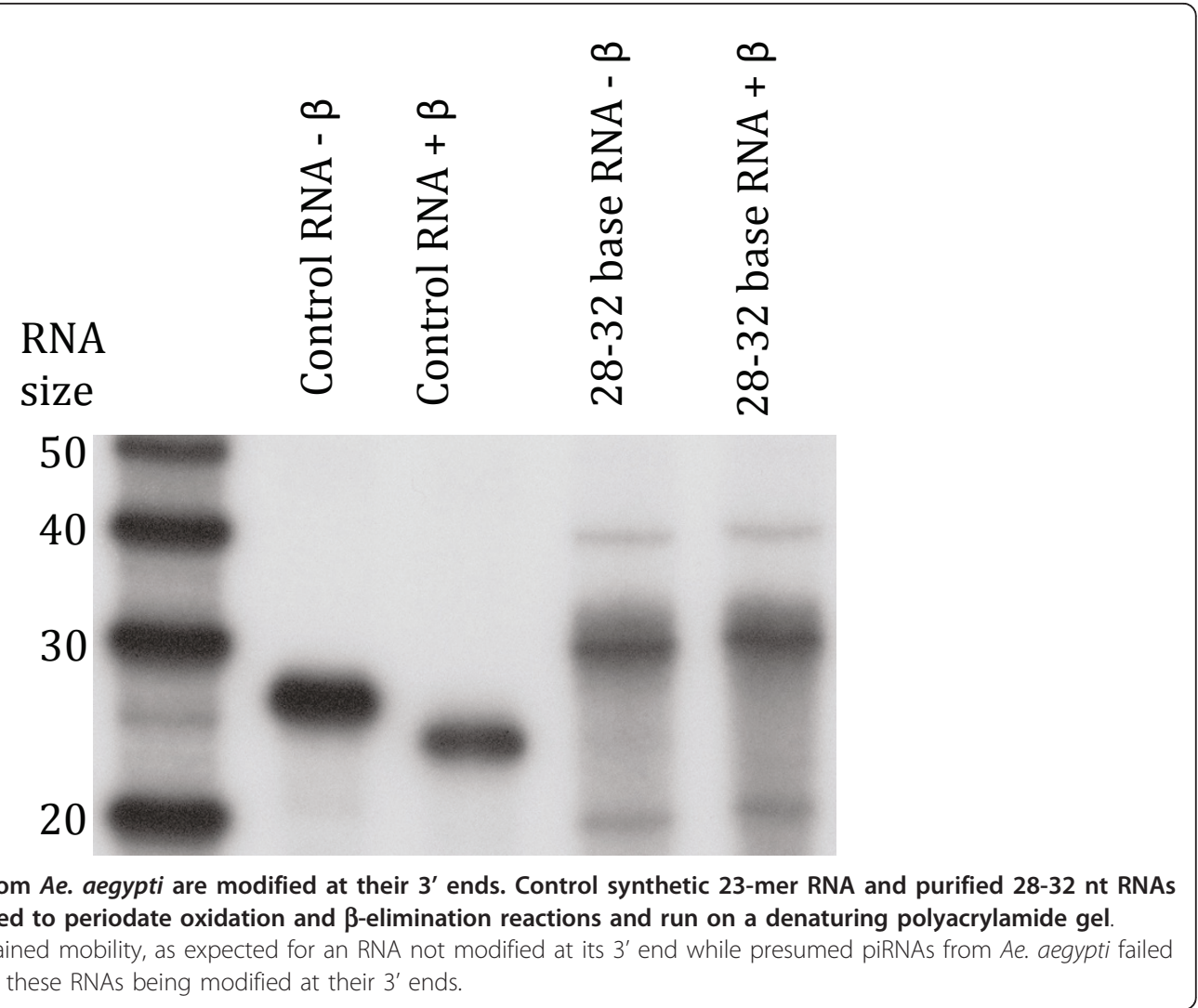

to determine if we could find the location of such clusters using our whole tissue libraries.

Restricting ourselves to uniquely mapping piRNAs (to unambiguously identify the origin of each piRNA in the genome), we first scanned all 4,758 Ae. aegypti supercontigs individually using a $5 \mathrm{~kb}$ sliding window and identified those windows that had ten or more piRNA sequences mapping to them. Identified windows were merged if they were found adjacent to each other. The boundaries of putative cluster loci were identified by scanning for the location of the furthest piRNA sequence on either end of the locus.

While this approach was similar to that used by Brennecke et al. (2007), it differed methodologically in two important ways. First, we did not collapse non-contiguous windows that were less than $20 \mathrm{~kb}$ apart. We reasoned that this should produce smaller but more well defined loci (i.e. with few regions of low piRNA density inside the cluster). Second, because of the much larger piRNA dataset available for Ae. aegypti, we assigned a cut-off of more than ten uniquely mapping piRNAs per window before making that window part of a cluster. Had this cut-off not been assigned, the resulting piRNAs clusters would cover a much larger portion of the genome, but would contain very few additional piRNAs. We validated this approach by applying it to our transgenic D. melanogaster library and compared the resulting piRNA cluster locations to published $D$. melanogaster clusters (Table 3)[6].

As expected many of the clusters defined by Brennecke et al. (2007) were found as two or more smaller clusters in our analysis. Nevertheless, there was overall a substantial amount of overlap between our analysis and these previously reported piRNA clusters. All but two of the top clusters were recovered (we did not recover clusters id11 and 15 reported by Brennecke et al. (2007)). A possible explanation may be that the piRNAs that map to these clusters are specific to germ line tissues and so were relatively underrepresented in our library. Nevertheless, because we were able to recover most of the same piRNA clusters as Brennecke et al. (2007) we considered our methodology sufficiently validated for analysis of our Ae. aegypti libraries.

\section{piRNA clusters in Ae. aegypti}

We used the seven Ae. aegypti libraries to independently identify piRNA clusters from each library. All seven analyses broadly agreed on the location of the top piRNA clusters on the Ae. aegypti supercontigs. Based on this broad agreement we combined the seven libraries into a single analysis (Table 4). The top 30 piRNA clusters (i.e. clusters containing the largest number of unique 


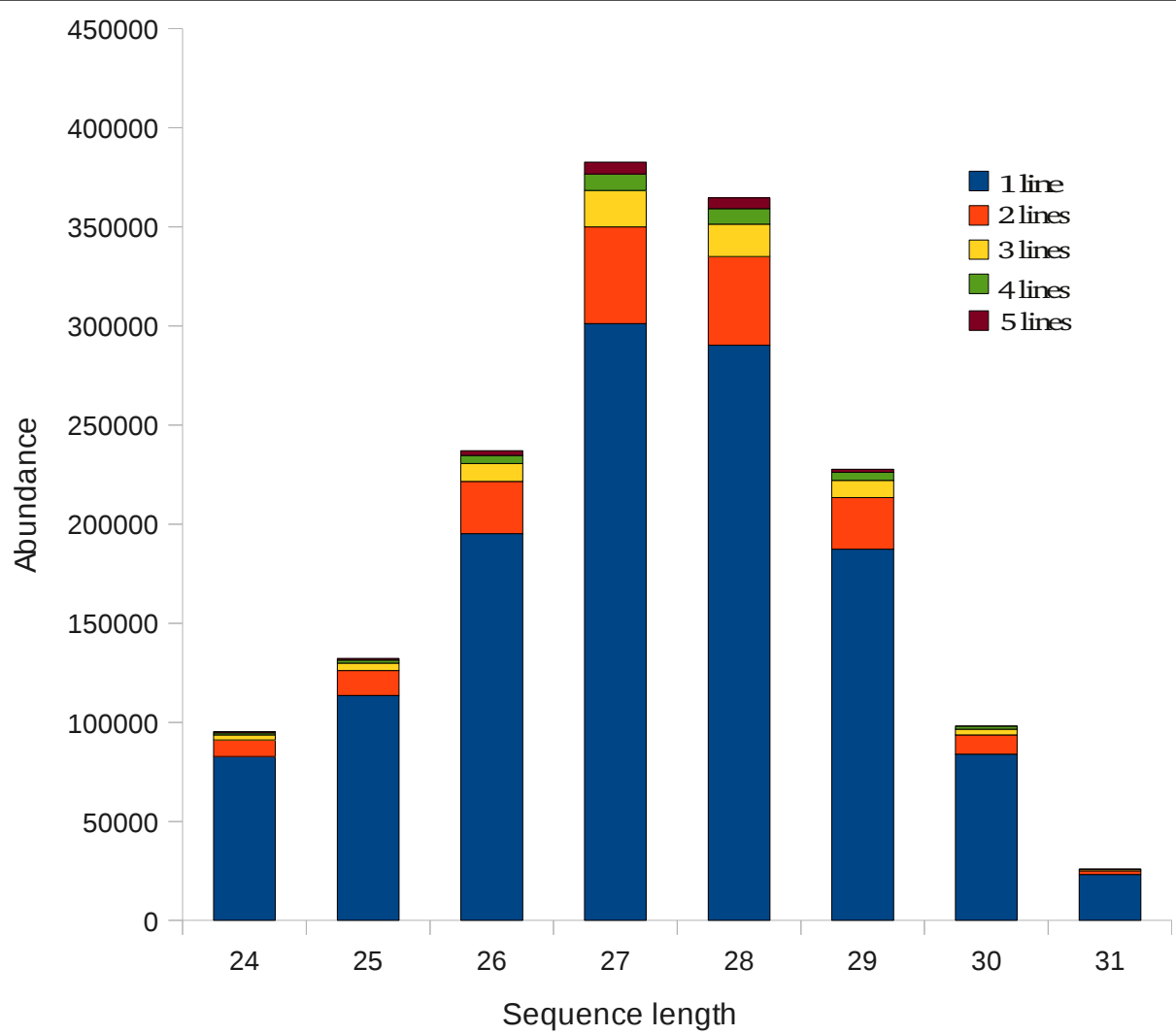

Figure 4 The abundance of piRNAs derived from five Ae. aegypti lines (libraries from replicate lines were collapsed) by size class. Each size class is divided into the number of piRNA sequences found in libraries derived from 1, 2, 3, 4, or 5 lines (the legend is shown at top right).

potential piRNA sequences) were supported by all seven Ae. aegypti libraries in rough proportion to the sequencing size of each library. Furthermore, $77 \%$ or more of the sequences mapping to the top 30 piRNA clusters were found in only one of the seven Ae. aegypti libraries, suggesting that different sequences in different libraries supported the same piRNA clusters.

The top 30 clusters ranged in size from 6 to $184 \mathrm{~kb}$ which a similar size range to those reported for $D$. melanogaster of 2 to $242 \mathrm{~kb}$ [6]. In Ae. aegypti the piRNA clusters occupied $20.6 \%$ of the assembled genome and could potentially generate $84 \%$ of the observed piRNAs. In comparison, D. melanogaster piRNA clusters have been reported to occupy only $3.5 \%$ of the genome and potentially produce $92 \%$ of the piRNAs [6]. The top 30 Ae. aegypti piRNA clusters were generally located either on different supercontigs or over $100 \mathrm{~kb}$ from each other on the same supercontig. The same analysis performed on our $D$. melanogaster library showed several piRNA clusters in closer physical proximity on the same chromosome (Table 3). This suggests that the Ae. aegypti piRNA clusters are more widespread and cover a greater proportional area of the genome than the clusters found in D. melanogaster. Many of the clusters, including the top 14, had piRNAs mapping predominantly to one strand. A similar bias was not observed when random genomic sequences of similar size to the clusters were examined (data not shown) suggesting that the observed bias was a characteristic of the individual clusters.

piRNA clusters have been suggested as being possible regulatory loci of transposons and were reported in $D$. melanogaster to consist of 70-99\% transposon sequences [6]. We did not observe such high proportions of transposon sequences in most Ae. aegypti clusters (Table 5). Comparison of Ae aegypti piRNA clusters with random Ae. aegypti genomic sequences of similar size to the clusters did not reveal a statistically significant pattern of transposon density or diversity inside piRNA clusters. However, we did observe that in many clusters transposon sequences were all, or nearly all, in the same orientation. This has also been previously observed in some D. melanogaster piRNA clusters [6]. If our presumed piRNA clusters were the sites of piRNA biogenesis it might be expected they should show increased levels of transcription. Fortunately, the results of an Ae. aegypti mRNAseq analysis were available on the VectorBase $A e$. aegypti genome browser (http://www.vectorbase.org) 
Table 3 The top 30 piRNA clusters in Ae. aegypti

\begin{tabular}{|c|c|c|c|c|c|c|c|c|c|c|c|c|c|c|}
\hline $\begin{array}{l}\text { Cluster } \\
\text { ID }\end{array}$ & Supercontig & start & stop & $\begin{array}{l}\text { cluster } \\
\text { size }\end{array}$ & $\begin{array}{l}\text { cluster } \\
\text { unique }\end{array}$ & $\begin{array}{l}\text { Distance to } \\
\text { nearest cluster }\end{array}$ & & $\begin{array}{l}\text { Relativ } \\
\text { quenc }\end{array}$ & $\begin{array}{l}\text { le pro } \\
\text { es ma }\end{array}$ & $\begin{array}{l}\text { portic } \\
\text { apping }\end{array}$ & $\begin{array}{l}\text { on of } \\
g \text { to } c\end{array}$ & $\begin{array}{l}\text { piRN } \\
\text { cluster }\end{array}$ & & $\begin{array}{l}\text { \% cluster reads } \\
\text { unique to one }\end{array}$ \\
\hline 1 & supercont1.478 & 490638 & 568444 & 78 & 116,897 & N/A & $23 \%$ & $10 \%$ & $20 \%$ & $10 \%$ & $9 \%$ & $20 \%$ & $9 \%$ & $77 \%$ \\
\hline 2 & supercont1.1 & 1151499 & 1278727 & 127 & 94,452 & 75 & $22 \%$ & $12 \%$ & $19 \%$ & $11 \%$ & $7 \%$ & $20 \%$ & $8 \%$ & $80 \%$ \\
\hline 3 & supercont1.286 & 1260644 & 1444752 & 184 & 89,740 & 113 & $25 \%$ & $9 \%$ & $23 \%$ & $8 \%$ & $8 \%$ & $19 \%$ & $7 \%$ & $82 \%$ \\
\hline 4 & supercont1.943 & 196392 & 264127 & 68 & 21,576 & N/A & $27 \%$ & $8 \%$ & $24 \%$ & $8 \%$ & $8 \%$ & $17 \%$ & $7 \%$ & $82 \%$ \\
\hline 5 & supercont1.209 & 235416 & 285783 & 50 & 19,243 & 36 & $26 \%$ & $10 \%$ & $23 \%$ & $9 \%$ & $8 \%$ & $17 \%$ & $7 \%$ & $82 \%$ \\
\hline 6 & supercont1.20 & 3110107 & 3224809 & 115 & 18,814 & 81 & $23 \%$ & $13 \%$ & $20 \%$ & $12 \%$ & $6 \%$ & $21 \%$ & $5 \%$ & $83 \%$ \\
\hline 7 & supercont1.402 & 860224 & 953549 & 93 & 17,519 & 181 & $22 \%$ & $13 \%$ & $20 \%$ & $12 \%$ & $6 \%$ & $21 \%$ & $5 \%$ & $83 \%$ \\
\hline 8 & supercont1.1145 & 61909 & 94822 & 33 & 13,440 & 38 & $25 \%$ & $11 \%$ & $22 \%$ & $9 \%$ & $8 \%$ & $20 \%$ & $5 \%$ & $80 \%$ \\
\hline 9 & supercont1.435 & 926765 & 972082 & 45 & 10,856 & 588 & $20 \%$ & $15 \%$ & $18 \%$ & $13 \%$ & $6 \%$ & $23 \%$ & $6 \%$ & $83 \%$ \\
\hline 10 & supercont1.379 & 15854 & 108077 & 92 & 9,550 & 285 & $23 \%$ & $13 \%$ & $21 \%$ & $13 \%$ & $6 \%$ & $21 \%$ & $4 \%$ & $85 \%$ \\
\hline 11 & supercont1.555 & 368190 & 374829 & 7 & 8,823 & 80 & $22 \%$ & $15 \%$ & $18 \%$ & $13 \%$ & $6 \%$ & $20 \%$ & $6 \%$ & $77 \%$ \\
\hline 12 & supercont1.209 & 305189 & 366980 & 62 & 7,140 & 40 & $24 \%$ & $10 \%$ & $22 \%$ & $10 \%$ & $7 \%$ & $22 \%$ & $5 \%$ & $83 \%$ \\
\hline 13 & supercont1.109 & 91700 & 129810 & 38 & 7,083 & 36 & $24 \%$ & $11 \%$ & $23 \%$ & $11 \%$ & $7 \%$ & $20 \%$ & $6 \%$ & $85 \%$ \\
\hline 14 & supercont1.697 & 504405 & 552871 & 48 & 6,382 & N/A & $30 \%$ & $6 \%$ & $28 \%$ & $7 \%$ & $9 \%$ & $15 \%$ & $6 \%$ & $85 \%$ \\
\hline 15 & supercont1.164 & 1210657 & 1248495 & 38 & 6,154 & 35 & $28 \%$ & $12 \%$ & $24 \%$ & $12 \%$ & $10 \%$ & $6 \%$ & $9 \%$ & $84 \%$ \\
\hline 16 & supercont1.83 & 1415166 & 1433185 & 18 & 5,799 & 74 & $19 \%$ & $14 \%$ & $17 \%$ & $13 \%$ & $8 \%$ & $22 \%$ & $7 \%$ & $83 \%$ \\
\hline 17 & supercont1.41 & 2610658 & 2644427 & 34 & 5,422 & 48 & $21 \%$ & $16 \%$ & $18 \%$ & $16 \%$ & $7 \%$ & $14 \%$ & $8 \%$ & $84 \%$ \\
\hline 18 & supercont1.2 & 275115 & 329629 & 55 & 4,663 & 42 & $18 \%$ & $18 \%$ & $16 \%$ & $18 \%$ & $6 \%$ & $17 \%$ & $6 \%$ & $86 \%$ \\
\hline 19 & supercont1.38 & 3097699 & 3148574 & 51 & 4,653 & 51 & $16 \%$ & $18 \%$ & $15 \%$ & $18 \%$ & $5 \%$ & $22 \%$ & $6 \%$ & $85 \%$ \\
\hline 20 & supercont1.192 & 1700025 & 1763125 & 63 & 4,631 & 44 & $18 \%$ & $18 \%$ & $14 \%$ & $19 \%$ & $6 \%$ & $20 \%$ & $7 \%$ & $86 \%$ \\
\hline 21 & supercont1.38 & 2995043 & 3039699 & 45 & 4,629 & 31 & $20 \%$ & $14 \%$ & $17 \%$ & $13 \%$ & $7 \%$ & $20 \%$ & $8 \%$ & $84 \%$ \\
\hline 22 & supercont1.109 & 1912714 & 1927564 & 15 & 4,339 & 395 & $19 \%$ & $9 \%$ & $12 \%$ & $8 \%$ & $18 \%$ & $17 \%$ & $17 \%$ & $92 \%$ \\
\hline 23 & supercont1.194 & 895141 & 974760 & 80 & 4,320 & 53 & $17 \%$ & $19 \%$ & $14 \%$ & $20 \%$ & $5 \%$ & $20 \%$ & $5 \%$ & $86 \%$ \\
\hline 24 & supercont1.226 & 725419 & 748642 & 23 & 4,280 & 93 & $21 \%$ & $14 \%$ & $17 \%$ & $13 \%$ & $7 \%$ & $21 \%$ & $7 \%$ & $84 \%$ \\
\hline 25 & supercont1.518 & 151663 & 158105 & 6 & 4,164 & 18 & $19 \%$ & $15 \%$ & $17 \%$ & $15 \%$ & $7 \%$ & $20 \%$ & $7 \%$ & $82 \%$ \\
\hline 26 & supercont1.38 & 2572763 & 2684616 & 112 & 4,119 & 69 & $17 \%$ & $19 \%$ & $14 \%$ & $20 \%$ & $5 \%$ & $19 \%$ & $6 \%$ & $87 \%$ \\
\hline 27 & supercont1.90 & 907728 & 931721 & 24 & 4,004 & 29 & $14 \%$ & $20 \%$ & $12 \%$ & $19 \%$ & $5 \%$ & $25 \%$ & $5 \%$ & $85 \%$ \\
\hline 28 & supercont1.2 & 1423334 & 1464722 & 41 & 3,928 & 41 & $20 \%$ & $15 \%$ & $17 \%$ & $14 \%$ & $7 \%$ & $21 \%$ & $7 \%$ & $86 \%$ \\
\hline 29 & supercont1.192 & 551266 & 584318 & 33 & 3,859 & 25 & $19 \%$ & $15 \%$ & $16 \%$ & $15 \%$ & $6 \%$ & $22 \%$ & $7 \%$ & $84 \%$ \\
\hline 30 & supercont1.1 & 1420282 & 1453931 & 34 & 3,827 & 39 & $24 \%$ & $13 \%$ & $21 \%$ & $13 \%$ & $6 \%$ & $16 \%$ & $6 \%$ & $84 \%$ \\
\hline
\end{tabular}

${ }^{1}$ Distances are from the center of one cluster to the next. Only cluster with 100 cluster unique piRNAs or more we considered. N/A indicates that no other piRNA cluster was found on this supercontig.

${ }^{2}$ In italics are the relative number of uniquely mapping piRNAs from each Ae. aegypti library. From left to right each number represents, library $1,2,4,6,10,11$, and 12.

${ }^{3}$ Percentage of all the reads mapping to the cluster that were derived from only one of the 7 Ae. aegypti libraries

[48]. By superimposing cluster and mRNAseq information on the genome browser we observed that most of our piRNA cluster locations appeared to overlap with increased numbers of mRNAseq sequences (Figure 5). While in some clusters increased mRNAseq numbers were confined to our piRNA cluster boundaries, other clusters appeared to have high transcription levels both inside and in areas adjacent to the cluster that may be evidence we have underestimated the size of some of the piRNA clusters in Ae. aegypti.

Several Ae. aegypti piRNA clusters overlapped with annotated genes. Many, but not all, of these gene sequences contained large numbers of piRNA sequences. Interestingly, those genes that mapped to many piRNA sequences were nearly all oriented in the opposite orientation to the majority of piRNAs in the cluster. Cluster genes that did not contain many piRNA sequences showed no particular orientation bias for their piRNA sequences.

\section{Ae. aegypti piRNAs and endo-siRNAs generated to endogenous transposons}

We combined the presumed piRNA sequences from all seven Ae. aegypti libraries into a single analysis (Table 
Table 4 Transposable element (TE) coverage and diversity in top Ae. aegypti piRNA clusters.

\begin{tabular}{|c|c|c|c|c|c|c|c|c|c|}
\hline $\begin{array}{l}\text { Cluster } \\
\text { ID }\end{array}$ & Supercontig & start & stop & $\begin{array}{l}\text { cluster TE } \\
\text { coverage }^{1}\end{array}$ & $\begin{array}{c}\text { mean TE } \\
\text { coverage of } \\
\text { random } \\
\text { sequences }^{2}\end{array}$ & $\begin{array}{c}\text { number of } \\
\text { standard } \\
\text { deviations, TE } \\
\text { coverage }^{3}\end{array}$ & $\begin{array}{c}\text { number of } \\
\mathrm{TE} \\
\text { elements } \\
\text { in cluster }^{4}\end{array}$ & $\begin{array}{l}\text { mean number of } \\
\text { TE elements in } \\
\text { random } \\
\text { sequences }\end{array}$ & $\begin{array}{c}\text { number of } \\
\text { standard } \\
\text { deviations, } \\
\text { number of } \\
\text { elements }\end{array}$ \\
\hline 1 & supercont1.478 & 490638 & 568444 & 0.226676263 & 0.419001465 & -1.416991717 & 38 & 61.235 & -1.304074578 \\
\hline 2 & supercont1.1 & 1151499 & 1278727 & 0.487436041 & 0.403024924 & 0.580732819 & 49 & 85.661 & -1.343104567 \\
\hline 3 & supercont1.286 & 1260644 & 1444752 & 0.395222395 & 0.396091577 & -0.005621894 & 64 & 107.978 & -1.210704338 \\
\hline 4 & supercont1.943 & 196392 & 264127 & 0.736078304 & 0.416404001 & 2.293783988 & 44 & 54.717 & -0.642122401 \\
\hline 5 & supercont1.209 & 235416 & 285783 & 0.410935515 & 0.419113904 & -0.058733961 & 24 & 43.614 & -1.499284524 \\
\hline 6 & supercont1.20 & 3110107 & 3224809 & 0.554179054 & 0.418089746 & 0.964456246 & 59 & 81.042 & -0.944476926 \\
\hline 7 & supercont1.402 & 860224 & 953549 & 0.402781647 & 0.411314707 & -0.059586613 & 42 & 69.042 & -1.264686072 \\
\hline 8 & supercont1.1145 & 61909 & 94822 & 0.391930486 & 0.417567435 & -0.178082295 & 22 & 30.692 & -0.897834546 \\
\hline 9 & supercont1.435 & 926765 & 972082 & 0.302241935 & 0.419841185 & -0.837861518 & 22 & 39.9 & -1.508018889 \\
\hline 10 & supercont1.379 & 15854 & 108077 & 0.611099063 & 0.409120133 & 1.411986233 & 50 & 69.013 & -0.893134363 \\
\hline 11 & supercont1.555 & 368190 & 374829 & 0 & 0.370578852 & -1.834660452 & 0 & 7.416 & -2.100509042 \\
\hline 12 & supercont1.209 & 305189 & 366980 & 0.459768255 & 0.423567623 & 0.271641456 & 22 & 51.899 & -2.088008606 \\
\hline 13 & supercont1.109 & 91700 & 129810 & 0.51195193 & 0.426534479 & 0.603357822 & 17 & 35.09 & -1.769148524 \\
\hline 14 & supercont1.697 & 504405 & 552871 & 0.498813626 & 0.428710684 & 0.485977742 & 30 & 42.633 & -0.960185323 \\
\hline 15 & supercont1.164 & 1210657 & 1248495 & 0.572980258 & 0.42009945 & 1.044389637 & 35 & 34.491 & 0.047763239 \\
\hline 16 & supercont1.83 & 1415166 & 1433185 & 0.648834628 & 0.409050447 & 1.485209324 & 16 & 18.324 & -0.361868136 \\
\hline 17 & supercont1.41 & 2610658 & 2644427 & 0.521261475 & 0.430863484 & 0.624645572 & 34 & 31.716 & 0.247718053 \\
\hline 18 & supercont1.2 & 275115 & 329629 & 0.446170779 & 0.422215779 & 0.174896759 & 53 & 46.562 & 0.478972045 \\
\hline 19 & supercont1.38 & 3097699 & 3148574 & 0.389653275 & 0.427616491 & -0.271821406 & 52 & 43.976 & 0.62870919 \\
\hline 20 & supercont1.192 & 1700025 & 1763125 & 0.514270772 & 0.419413566 & 0.675173572 & 59 & 52.334 & 0.430866518 \\
\hline 21 & supercont1.38 & 2995043 & 3039699 & 0.538146315 & 0.428465514 & 0.764356465 & 40 & 39.557 & 0.037258641 \\
\hline 22 & supercont1.109 & 1912714 & 1927564 & 0.013803784 & 0.405080673 & -2.303191497 & 5 & 15.475 & -1.787236268 \\
\hline 23 & supercont1.194 & 895141 & 974760 & 0.510474755 & 0.421488238 & 0.642327725 & 64 & 62.082 & 0.1058288 \\
\hline 24 & supercont1.226 & 725419 & 748642 & 0.575051671 & 0.411773027 & 1.040816358 & 25 & 22.408 & 0.332107048 \\
\hline 25 & supercont1.518 & 151663 & 158105 & 0.458637281 & 0.360901428 & 0.487675463 & 14 & 7.232 & 1.906352115 \\
\hline 26 & supercont1.38 & 2572763 & 2684616 & 0.49881095 & 0.402104083 & 0.653081355 & 87 & 78.081 & 0.349121968 \\
\hline 27 & supercont1.90 & 907728 & 931721 & 0.420813537 & 0.422340266 & -0.010037376 & 26 & 23.628 & 0.304378812 \\
\hline 28 & supercont1.2 & 1423334 & 1464722 & 0.508492595 & 0.4232601 & 0.598461412 & 35 & 37.239 & -0.202802747 \\
\hline 29 & supercont1.192 & 551266 & 584318 & 0.405984328 & 0.414953558 & -0.060505091 & 31 & 30.782 & 0.02131827 \\
\hline 30 & supercont1.1 & 1420282 & 1453931 & 0.61961367 & 0.423640435 & 1.324250019 & 26 & 31.445 & -0.541018989 \\
\hline
\end{tabular}

${ }^{1}$ Proportion the cluster with similarity to known Ae. aegypti TE sequences. Data derived from running the program RepeatMasker.

21000 random sequences of the same bp. size as the cluster were used.

${ }^{3}$ Number of standard deviations between the the observed TE coverage and the mean TE coverage of random sequences

${ }^{4}$ Number of different kinds of TE sequences found in the cluster

5) and mapped these to annotated Ae. aegypti transposons in the RepBase and TEfam databases [49] (http:// tefam.biochem.vt.edu/) (Additional File 2, Table S1). In our D. melanogaster library, almost $50 \%$ of presumptive unique piRNAs mapped to transposon sequences, slightly higher than the $42 \%$ seen for the $23-29$ nt fraction obtained from $D$. melanogaster Oregon $\mathrm{R}$ total RNA [31]. In contrast, despite having a larger transposable element load than D. melanogaster, only $19.48 \%$ of the presumed piRNAs mapped to annotated transposable elements. It is entirely possible that this lower value may be in part due to unannoted transposable element sequences present in the Ae. aegypti reference genome. The majority (89.75\%) of transposon-specific Ae. aegypti piRNAs mapped to class I transposons, $5.95 \%$ to class II transposons, $2.03 \%$ to MITEs and $2.27 \%$ to other transposons that currently cannot be easily assigned (Table 2 and Table S1). This reflects to some degree the relative abundance of the class I and class II transposons (without MITEs) in the Ae. aegypti reference genome (57\% class I, 6.5\% class II, 34.4\% MITEs, 2.2\% Helitrons [16]). Two LTR retrotransposons, Ty3_gypsy and $\mathrm{Pao} \_\mathrm{Bel}$ 
Table 5 Number and percent abundance of piRNA and siRNA sequences in Ae. aegypti and D. melanogaster libraries, as well piRNA and siRNA sequences matching known TE sequences

\begin{tabular}{|c|c|c|c|c|c|c|c|c|}
\hline \multirow{3}{*}{$\begin{array}{l}\text { piRNA } \\
\text { Total }\end{array}$} & \multicolumn{4}{|c|}{ Ae. aegypti } & \multicolumn{4}{|c|}{ D. melanogaster } \\
\hline & \multicolumn{2}{|c|}{ number of piRNA sequences } & \multicolumn{2}{|c|}{ percent of piRNAs } & \multicolumn{2}{|c|}{ number of piRNA sequences } & \multicolumn{2}{|c|}{ percent of piRNAs } \\
\hline & $11,173,973$ & $(5,860,037)$ & $100.00 \%$ & $(100.00 \%)$ & 271,626 & $(205,307)$ & $100.00 \%$ & $(100.00 \%)$ \\
\hline TE & $2,177,176$ & $(1,240,908)$ & $19.48 \%$ & $(21.18 \%)$ & 135,387 & $(110,068)$ & $49.84 \%$ & $(53.61 \%)$ \\
\hline Retrotransposons & $1,987,059$ & $(1,114,018)$ & $17.78 \%$ & $(19.01 \%)$ & 129,418 & $(105,485)$ & $47.65 \%$ & (51.38\%) \\
\hline DNA transposons & 113,030 & $(73,584)$ & $1.01 \%$ & $(1.26 \%)$ & 5,969 & $(4,583)$ & $2.20 \%$ & $(2.23 \%)$ \\
\hline MITE & 29,558 & $(25,324)$ & $0.26 \%$ & $(0.43 \%)$ & $N / A^{2}$ & $\mathrm{~N} / \mathrm{A}$ & $\mathrm{N} / \mathrm{A}$ & $\mathrm{N} / \mathrm{A}$ \\
\hline other $^{1}$ & 47,529 & $(27,982)$ & $0.43 \%$ & $(0.48 \%)$ & N/A & $\mathrm{N} / \mathrm{A}$ & $\mathrm{N} / \mathrm{A}$ & $\mathrm{N} / \mathrm{A}$ \\
\hline Endo-siRNA & \multicolumn{2}{|c|}{ number of siRNA sequences } & \multicolumn{2}{|c|}{ percent of siRNAs } & \multicolumn{2}{|c|}{ number of siRNA sequences } & \multicolumn{2}{|c|}{ percent of siRNAs } \\
\hline Total & 765,132 & $(446,834)$ & $100.00 \%$ & $(100.00 \%)$ & 86,620 & $(54,049)$ & $100.00 \%$ & $(100.00 \%)$ \\
\hline$\overline{T E}$ & 216,859 & $(154,465)$ & $28.34 \%$ & (34.57\%) & 15,870 & $(13,280)$ & $18.32 \%$ & $(24.57 \%)$ \\
\hline Retrotransposons & 133,042 & $(100,067)$ & $17.39 \%$ & (22.39\%) & 15,276 & $(12,720)$ & $17.64 \%$ & (23.53\%) \\
\hline DNA transposons & 28,578 & $(18,692)$ & $3.74 \%$ & $(4.18 \%)$ & 594 & $(560)$ & $0.69 \%$ & $(1.04 \%)$ \\
\hline MITE & 32,623 & $(23,667)$ & $4.26 \%$ & $(5.30 \%)$ & N/A & N/A & $\mathrm{N} / \mathrm{A}$ & $\mathrm{N} / \mathrm{A}$ \\
\hline other $^{1}$ & 22,616 & $(12,039)$ & $2.96 \%$ & (2.69\%) & $\mathrm{N} / \mathrm{A}$ & $\mathrm{N} / \mathrm{A}$ & $\mathrm{N} / \mathrm{A}$ & $\mathrm{N} / \mathrm{A}$ \\
\hline
\end{tabular}

Numbers in parentheses exclude duplicate sequences.

${ }^{1}$ TE elements such as Penelope that cannot be put into any of the other categories.

${ }^{2}$ Indicates that no elements from these categories have been reported in $D$. melanogaster.

accounted for over $57 \%$ of all transposon-specific piRNAs with almost 44\% mapping to the Ty3_gypsy retrotransposon (Additional File 2, Table S1). The number of unique piRNAs generated to MITEs is, however, proportionally less than their abundance in the genome. As described below, MITEs appear to be more preferentially targeted by endo-siRNAs and the relative lack of coding sequences may explain why proportionally few piRNAs map to them.

We generated piRNA density maps to seven $A e$. aegypti transposable elements (Additional File 3, Figure S1). The class II transposon AeBuster1 is a member of the $h A T$ superfamily and is active in interplasmid transposition assays performed in Ae. aegypti and D. melanogaster embryos [50]. Four intact copies are present in the genome reference strain. AeTango 2 is also a class II transposon but belongs to the IS630-Tc1-mariner superfamily, is present in 25 copies in the Ae. aegypti genome of which one copy is possibly an active element based on bioinformatic properties (an intact transposase gene open reading frame, intact terminal inverted repeats, and the presence of the TA target site duplication) [51]. Juan-A (called jockey Ele1 in Tefam (http://tefam.biochem.vt.edu/)) is a non-LTR retrotransposon, is a member of the jockey clade, comprises approximately $3 \%$ of the entire genome sequence and is widely distributed amongst mosquito species in which it has been proposed to have been recently active [52]. Lian non-LTR retrotransposons (called LOA in Tefam) have been estimated to be present in the Ae. aegypti (Rockefeller) strain from approximately 460-1,380 copies per haploid genome based on the analysis of a genomic library. [53]. MosquI, (called I Ele1 in Tefam) is also a non-LTR retrotransposon, is a member of the $I$ clade and, based on analysis of the same genomic library, is believed to be present in low copies [54]. Pao_Bel-ele1 and Ty3_gypsy_ele1 are both LTR retrotransposons with the former being present in 11 copies (four being full length) and

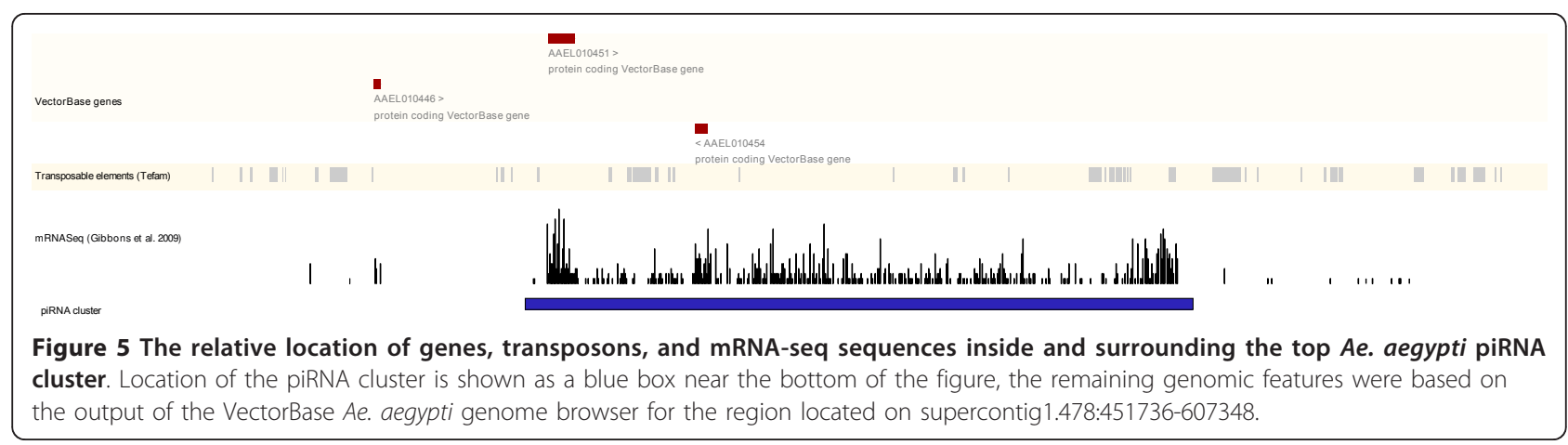


the later being present in nine copies (four full length) [16] (http://tefam.biochem.vt.edu/). As described above, together these LTR retrotransposons account for almost $58 \%$ of the piRNAs that target transposons in the Ae. aegypti genome. All seven transposable elements were targeted by piRNAs and all but MosquI-Aa2 contained at least one ping-pong signature overlap within their transcripts implying that they could be subject to recognition and silencing by this pathway (Additional File 3, Figure S1). piRNAs mapped to both strands for all seven transposable elements but there was a strong antisense bias observed for the AeTango 2 and Ty3_gypsy_Ele1 transposable elements and especially for the Juan-A non-LTR retrotransposon. These piRNA density maps of Ae. aegypti transposons are consistent with what has been found for $D$. melanogaster transposons $[6,9,35]$

In D. melanogaster endo-siRNAs have been shown to target transposons both in the germ line and the soma [10,55-57]. We investigated if this was also the case in Ae. aegypti by analyzing $21 \mathrm{nt}$ long sequences in our libraries. In our D. melanogaster library $18 \%$ of sequences matched known transposons, a number slightly lower than a previous estimate of transposons in D. melanogaster cell cultures (27\%, [55]) (Table 2). In the Ae. aegypti libraries $28 \%$ of sequences matched known transposons.

Because we used libraries derived from whole mosquitoes we were not able to distinguish between somatic and germ line-specific siRNA sequences. Nevertheless, it appeared likely that a large percentage of Ae. aegypti endo-siRNAs participate in interactions with transposons and could therefore be hypothesized to be at least partially involved in their regulation. Furthermore, it is interesting to note that a large number Ae. aegypti endo-siRNAs mapped to miniature inverted terminal element (MITE) sequences (Table 5). MITEs have, by definition, no coding potential and no such elements have been described from D. melanogaster. However, MITEs make up a substantial percentage (16\%) of the assembled Ae aegypti genome [16], but to date little is known about their transcription or regulation by the host genome. The substantially larger percentage of endo-siRNAs mapping to MITEs (15.03\% of endo-siRNAs mapping to transposon sequences) than piRNAs $(2.03 \%)$ may have its basis in their regulation or, more likely, arise from the production of foldback dsRNAs generated from the unidirectional transcription across the MITE terminal inverted repeats.

\section{piRNAs generated to introduced transposon sequences}

The seven Ae. aegypti libraries included six libraries derived from mosquito lines that were germ line transformed with the piggyBac, Mos1 and Hermes transposons (Table 1). We attempted to detect the activation of a silencing mechanism targeted specifically at these introduced sequences by looking for small RNAs derived from them. We limited our analysis to piRNAs because siRNAs (21 nt) were considered too small to be reliably mapped for this analysis. In order to maximize the chances that any identified piRNA sequence had originated from the introduced sequences rather than from the host genome we ignored any piRNA sequence that mapped to the Ae. aegypti genome assembly and did not allow any mismatches between the piRNA and introduced sequences (Table 6, Figure 6). The analysis was slightly complicated by the fact that some of the transformation plasmids had regions of identical sequences, allowing piRNAs to appear to be derived from more than one plasmid. For example, library 1 was derived from a mosquito line transformed with the plasmid pMos3DB2Her; six piRNA sequences were found to match pMos3DB2Her, but also two piRNAs matched the plasmid autoHermes. However, these last two piRNAs were the same as we found to map to pMos3DB2Her and are therefore likely to be artifacts. Such likely artifact mapping events are indicated in Table 6 with parentheses.

piRNAs were found to map both to transposon and non-transposon portions of introduced sequences (Figure 6). While the numbers were very small there appeared to be more piRNAs mapping to pMos3DB2Her in libraries 1 and 2 than in other libraries, suggesting that at least some of these piRNAs were indeed derived from the transformed sequence. We compared these to the presumptive piRNAs generated to Hermes from a small RNA library we constructed from its natural host, the housefly Musca domestica (Figure 7). While the number and types of piRNAs were far larger in housefly, two of these piRNAs were also generated in the transgenic Ae. aegypti line. piRNAs to Hermes were also generated to the transgenic lines containing an autonomous Hermes transposon (data not shown). This suggests that new piRNAs have been generated to introduced sequences sometime within the approximate 35 mosquito generations that separated the introduction of the transformation plasmids into the germ line and collection for library construction.

\section{piRNA generating genes}

As described above, analysis of our Ae. aegypti libraries indicated that most transposon associated piRNA sequences were predominantly found within gene boundaries. Previous studies have described associations of individual genes with high numbers of piRNA sequences mapping to these genes [58-60]. We sought to identify such genes in Ae. aegypti by identifying genes sequences (defined here as including all annotated UTR, 
Table 6 Number of piRNA and siRNA sequences that align to transposon sequences transformed into mosquito germlines.1

\begin{tabular}{|c|c|c|c|c|c|c|c|}
\hline piRNA & 1 & 4 & 2 & 6 & 11 & 12 & 10 \\
\hline template $^{2}$ & \multicolumn{2}{|c|}{ pMos3DB2Her } & \multicolumn{2}{|c|}{ pBac3EB2Mos } & autohermes & pMos3DBhspPBac & Wild \\
\hline pMos3DB2Her 3,175 $(1,839)$ bp. & $1(1)$ & $7(4)^{3}$ & 0 & 0 & 0 & 0 & $2(1)$ \\
\hline pBac3EB2Mos 3,520 $(1,038) \mathrm{bp}$ & 0 & 0 & 0 & 0 & 0 & 0 & 0 \\
\hline autohermes $3,081(1,830) \mathrm{bp}$. & 0 & 0 & 0 & 0 & 0 & 0 & 0 \\
\hline pMos3DBhspPBac $3,081(1,785)$ bp. & 0 & 0 & 0 & 0 & 0 & 0 & 0 \\
\hline \multicolumn{8}{|l|}{ siRNA } \\
\hline pMos3DB2Her 3,175 $(1,839)$ bp. & 0 & 0 & 0 & 0 & 0 & 0 & 0 \\
\hline pBac3EB2Mos 3,520 $(1,038) \mathrm{bp}$. & 0 & 0 & $1(1)$ & $1(1)$ & 0 & 0 & 0 \\
\hline autohermes $3,081(1,830) \mathrm{bp}$. & 0 & 0 & 0 & 0 & 0 & 0 & 0 \\
\hline pMos3DBhspPBac 3,081 $(1,785)$ bp. & 0 & 0 & 0 & 0 & 0 & 0 & 0 \\
\hline
\end{tabular}

${ }^{1}$ All libraries but library \#10 were derived from Ae. aegypti lines containing germline transformations of transposon sequences, the name of the transformation plasmid is indicated below each library number. Only matches to the transposon derived portions of the transformation plasmid are reported. Sequences that also match the Ae. aegypti genome assembly are excluded

${ }^{2}$.Transposon sequence alignment template. Transposon sequences from these plasmids were used as templates for library alignments. Indicated next to each plasmid name is the size (bp) of the transposon derived sequences and in parentheses the size of the transposase sequence.

${ }^{3}$ Marked in bold are numbers where the mosquito line is transformed with the alignment template sequence. Numbers in parentheses indicate the number of alignments to the transposase.

intron, and exon regions) with the highest density of uniquely mapping piRNAs. Because many Ae. aegypti genes have yet to be annotated with specific gene functions we attempted to assign a probable function to them by searching several databases (VectorBase,
SwissProt, and NCBI) for similar genes with known functions (Table 7).

While this is a preliminary overview of the piRNAgene association in Ae. aegypti, several features stand out. Among the top 30 genes eight have strong

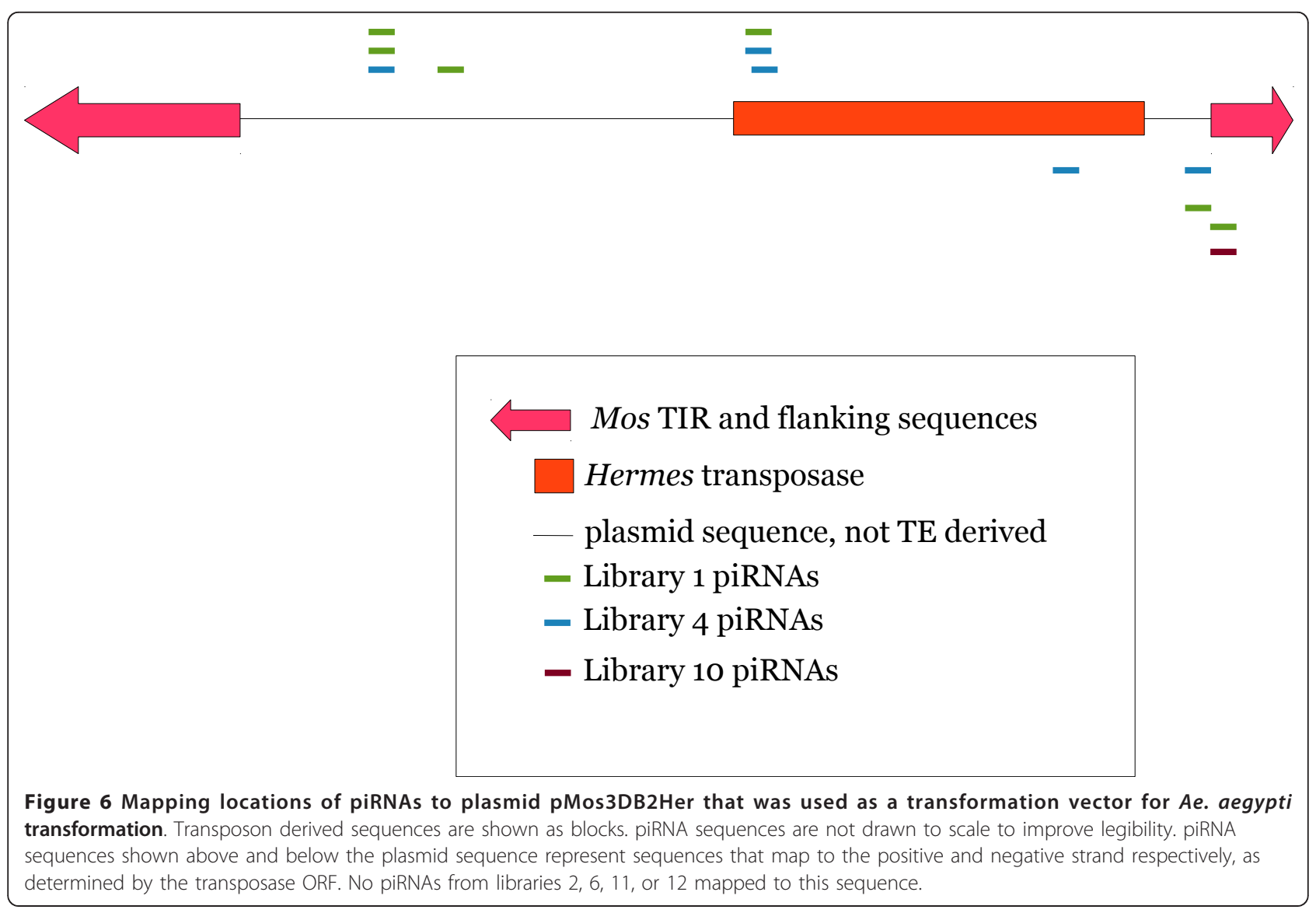




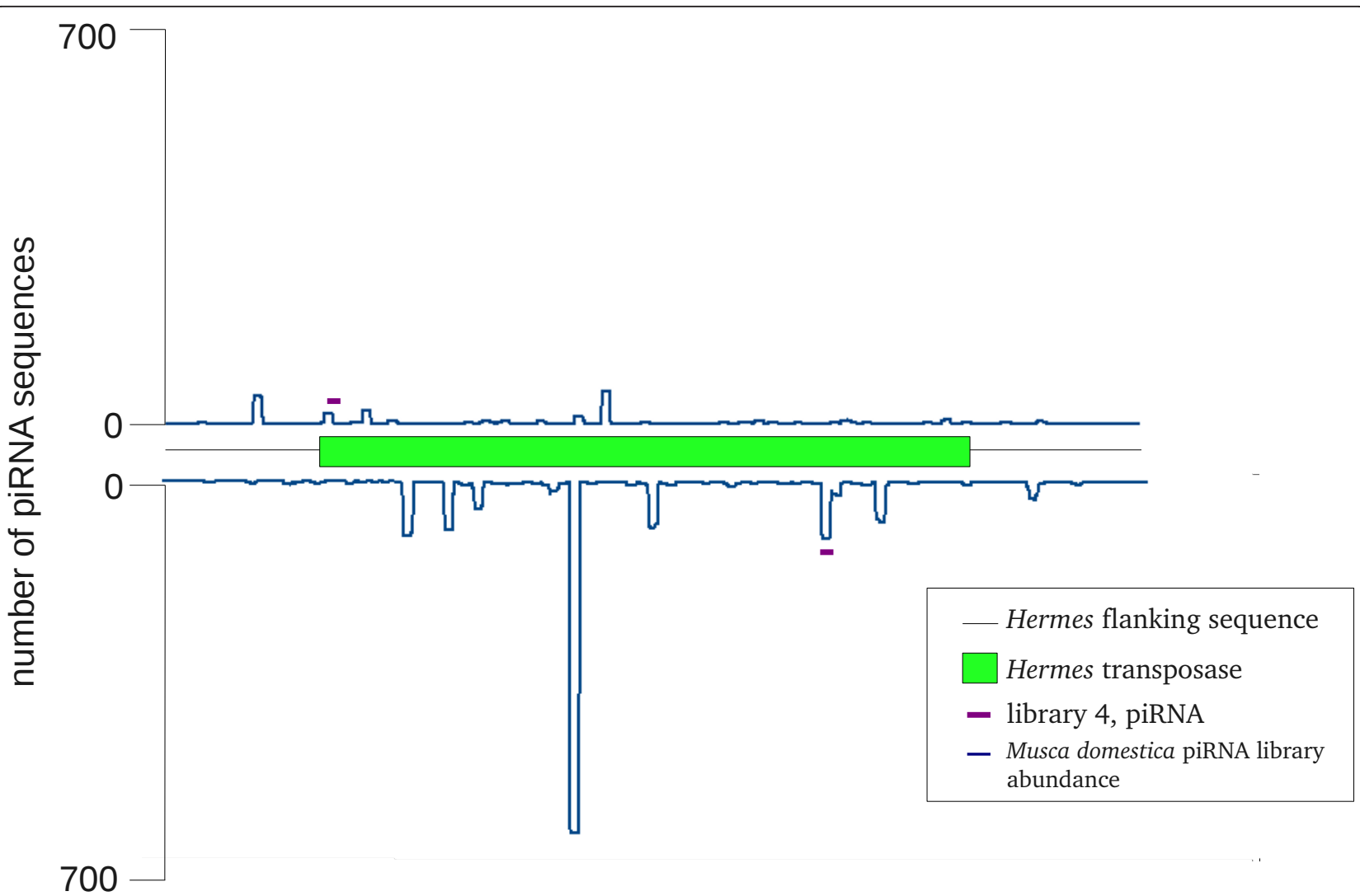

Figure 7 Location of where two Ae. aegypti piRNA sequences from library 4 map to the $M$. domestica Hermes transposase. Also shown is the abundance of piRNA sequences mapping to the sense and antisense strands of the Hermes transposon from a M. domestica small RNA library (abundance scales for both strands are shown on the left).

similarities to viral-derived sequences with all of them having the majority of their piRNAs mapping to the antisense strand. While several previous studies in mosquitoes have linked silencing of viral transcripts with 21 nt viRNAs, a definitive link between piRNAs and viral sequences in mosquitoes has yet to be well established [61-63]. Two Ae. aegypti genes in Table 7 have similarities to genes previously associated with possible transposon regulation. Gene AAEL001004 appears to be an Ae. aegypti homolog of the D. melanogaster maelstrom gene. Maelstrom localizes to the nuage and has been implicated in transposon regulation in mice and D. melanogaster [64-66]. The second gene of interest is AAEL007686 that has sequence similarities with the MafB transcription factor and is a homolog of the $D$. melanogaster traffic jam gene. Traffic jam has been described as a piRNA cluster, with most of the piRNAs arising from the sense strand of its 3' UTR [60]. While the Ae. aegypti gene AAEL007686 could possibly give rise to at least 2,439 piRNAs (Table 7) it was not found inside an identified piRNA cluster. The great majority of piRNAs mapped to the sense strand of AAEL007686 but, in contrast to D. melanogaster traffic jam, piRNAs mapped predominantly to the 3 ' end of the open reading frame rather than to the UTR (Figure 8). 98.3\% of piRNAs mapping to the Ae. aegypti tj gene contain $\mathrm{U}$ in their first position while $75.3 \%$ commenced with the 25 nt sequence 5' UAUUGACAACAGAAGUAACGAAUGA 3' with most variations being in the small number of additional ribonucleotides present at the 3' end. We confirmed using 3'RACE that the actual transcription termination site of this gene was consistent with its annotation and also confirmed, using RNAseq data, that this piRNA site was located in the translated part of the transcript (Additional File 4, Figure S1). We also examined the piRNA density of the D. melanogaster tj gene using our own $D$. melanogaster library and published D. melanogaster piRNA libraries $[6,46]$ and confirmed the previous location of the majority of these to the sense strand of the 3' UTR (Figure 8)[60]. Interestingly, the Ae. aegypti maelstrom piRNAs all map to the 3' UTR of the sense strand, which we also confirmed by RNAseq analysis of this gene (Figure 9, Additional File 5, Figure S1). $88.6 \%$ of these piRNAs contained A at the $10^{\text {th }}$ position of the piRNA. We examined our own and published $D$. melanogaster libraries $[6,46]$ for piRNAs 
Table 7 The top 30 Ae. aegypti genes with highest piRNA density

\begin{tabular}{|c|c|c|c|c|c|c|c|c|}
\hline Gene & $\begin{array}{l}\text { Gene } \\
\text { length } \\
\text { (bps.) }\end{array}$ & $\begin{array}{l}\text { Unique } \\
\text { mapping } \\
\text { piRNAs }\end{array}$ & $\begin{array}{l}\text { piRNA density } \\
\text { (piRNAs/gene } \\
\text { bps.) }\end{array}$ & $\begin{array}{l}\text { strand } \\
\text { percent } \\
+/-\end{array}$ & $\begin{array}{l}\text { gene inside } \\
\text { piRNA } \\
\text { cluster }\end{array}$ & $\begin{array}{l}\text { Function of best match (if } \\
\text { available) and species }\end{array}$ & $\begin{array}{l}\text { Best } \\
\text { match } \\
\text { database }\end{array}$ & $\begin{array}{l}\text { Best match } \\
\text { accession }\end{array}$ \\
\hline AAEL011224 & 303 & 16488 & 54.6 & $100 / 0$ & yes & hypothetical protein & VectorBase & AAEL011224 \\
\hline AAEL007866 & 1334 & 10651 & 8.0 & $99 / 1$ & yes & putative NS1 protein, Aedes flavivirus & NR & YP_003084126 \\
\hline AAEL007861 & 1182 & 4356 & 3.7 & $100 / 0$ & yes & $\begin{array}{l}\text { uncharacterized transmembrane } \\
\text { protein DDB_G0289901, } \\
\text { Dictyostelium discoideum }\end{array}$ & Swiss-Prot & Y8625_DICDI \\
\hline AAEL010454 & 1425 & 4084 & 2.9 & $90 / 10$ & yes & hypothetical protein & VectorBase & AAEL010454 \\
\hline AAEL000120 & 1020 & 1432 & 1.4 & $94 / 6$ & no & $\begin{array}{l}\text { nucleoprotein } \mathrm{N} \text {, bovine ephemeral } \\
\text { fever virus }\end{array}$ & Swiss-Prot & NCAP_BEFV \\
\hline AAEL009005 & 629 & 856 & 1.4 & $100 / 0$ & no & adult cuticle protein, Aedes aegypti & VectorBase & AAEL009005 \\
\hline AAEL005277 & 4038 & 4891 & 1.2 & $100 / 0$ & yes & $\begin{array}{l}\text { Zinc finger protein on ecdysone } \\
\text { puffs, Drosophila melanogaster }\end{array}$ & Swiss-Prot & PEP_DROME \\
\hline AAEL009525 & 1236 & 1494 & 1.2 & $100 / 0$ & no & $\begin{array}{l}\text { nucleoprotein putative, Aedes } \\
\text { aegypti }\end{array}$ & VectorBase & AAEL009525 \\
\hline AAEL005213 & 1377 & 1582 & 1.1 & $100 / 0$ & no & hypothetical protein & VectorBase & AAEL005213 \\
\hline AAEL000668 & 1333 & 1522 & 1.1 & $100 / 0$ & no & conserved hypothetical protein & VectorBase & AAEL000668 \\
\hline AAEL001004 & 6251 & 7009 & 1.1 & $100 / 0$ & no & $\begin{array}{l}\text { protein maelstrom homolog, Aedes } \\
\text { aegypti }\end{array}$ & Swiss-Prot & MAEL_AEDAE \\
\hline AAEL007686 & 2253 & 2439 & 1.1 & $100 / 0$ & no & $\begin{array}{l}\text { Transcription factor MafB, Coturnix } \\
\text { coturnix jopnica }\end{array}$ & Swiss-Prot & MAFB_COTJA \\
\hline AAEL001772 & 2562 & 2643 & 1.0 & $100 / 0$ & no & $\begin{array}{l}\text { RNA-dependent RNA polymerase, } \\
\text { Nyamanini virus }\end{array}$ & NR & YP_002905337 \\
\hline AAEL005456 & 966 & 841 & 0.9 & $100 / 0$ & yes & $\begin{array}{l}\text { nucleoprotein putative, Aedes } \\
\text { aegypti }\end{array}$ & VectorBase & AAEL005456 \\
\hline AAEL000808 & 978 & 811 & 0.8 & $100 / 0$ & yes & $\begin{array}{l}\text { nucleoprotein putative, Aedes } \\
\text { aegypti }\end{array}$ & VectorBase & AAEL000808 \\
\hline AAEL013013 & 1088 & 862 & 0.8 & $100 / 0$ & no & hypothetical protein & VectorBase & AAEL013013 \\
\hline AAEL006843 & 21911 & 17268 & 0.8 & $100 / 0$ & no & $\begin{array}{l}\text { NEDD4-binding protein 2, Homo } \\
\text { sapiens }\end{array}$ & Swiss-Prot & N4BP2_HUMAN \\
\hline AAEL006159 & 330 & 247 & 0.8 & $100 / 0$ & yes & Peritrophin-1, Anopheles gambiae & Swiss-Prot & PE1_ANOGA \\
\hline AAEL005225 & 762 & 570 & 0.7 & $100 / 0$ & no & conserved hypothetical protein & VectorBase & AAEL005225 \\
\hline AAEL004959 & 1242 & 914 & 0.7 & $100 / 0$ & no & $\begin{array}{l}\text { nucleoprotein putative, Aedes } \\
\text { aegypti }\end{array}$ & VectorBase & AAEL004959 \\
\hline AAEL009873 & 1602 & 1142 & 0.7 & $78 / 22$ & no & $\begin{array}{l}\text { nucleoprotein, bovine ephemeral } \\
\text { fever virus }\end{array}$ & Swiss-Prot & NCAP_BEFV \\
\hline AAEL005768 & 738 & 511 & 0.7 & $100 / 0$ & no & hypothetical protein & VectorBase & AAEL005768 \\
\hline AAEL007499 & 579 & 372 & 0.6 & $100 / 0$ & no & hypothetical protein & VectorBase & AAEL007499 \\
\hline AAEL007844 & 2572 & 1490 & 0.6 & $100 / 0$ & no & $\begin{array}{l}\text { glycoprotein, spring viremia of carp } \\
\text { virus }\end{array}$ & NR & ABB13496 \\
\hline AAEL010887 & 60098 & 34174 & 0.6 & $100 / 0$ & no & $\begin{array}{l}\text { Myosin heavy chain } \mathrm{IB}_{1} \\
\text { Acanthamoeba castellanii }\end{array}$ & Swiss-Prot & MYSB_ACACA \\
\hline AAEL000673 & 1461 & 753 & 0.5 & $100 / 0$ & no & $\begin{array}{l}\text { arbohydrate kinase domain- } \\
\text { containing protein, Mus musculus }\end{array}$ & Swiss-Prot & CARKD_MOUSE \\
\hline AAEL000276 & 71446 & 36638 & 0.5 & $1 / 99$ & no & $\begin{array}{l}\text { VPRBP protein, Culex } \\
\text { quinquefasciatus }\end{array}$ & $\mathrm{NR}$ & XP_001865214 \\
\hline AAEL012346 & 1177 & 598 & 0.5 & $100 / 0$ & no & hypothetical protein & VectorBase & AAEL012346 \\
\hline AAEL006704 & 1356 & 684 & 0.5 & $100 / 0$ & no & $\begin{array}{l}\text { fibrinogen and fibronectin, Aedes } \\
\text { aegypti }\end{array}$ & VectorBase & AAEL006704 \\
\hline AAEL003715 & 615 & 298 & 0.5 & $100 / 0$ & no & hypothetical protein & VectorBase & AAEL003715 \\
\hline
\end{tabular}



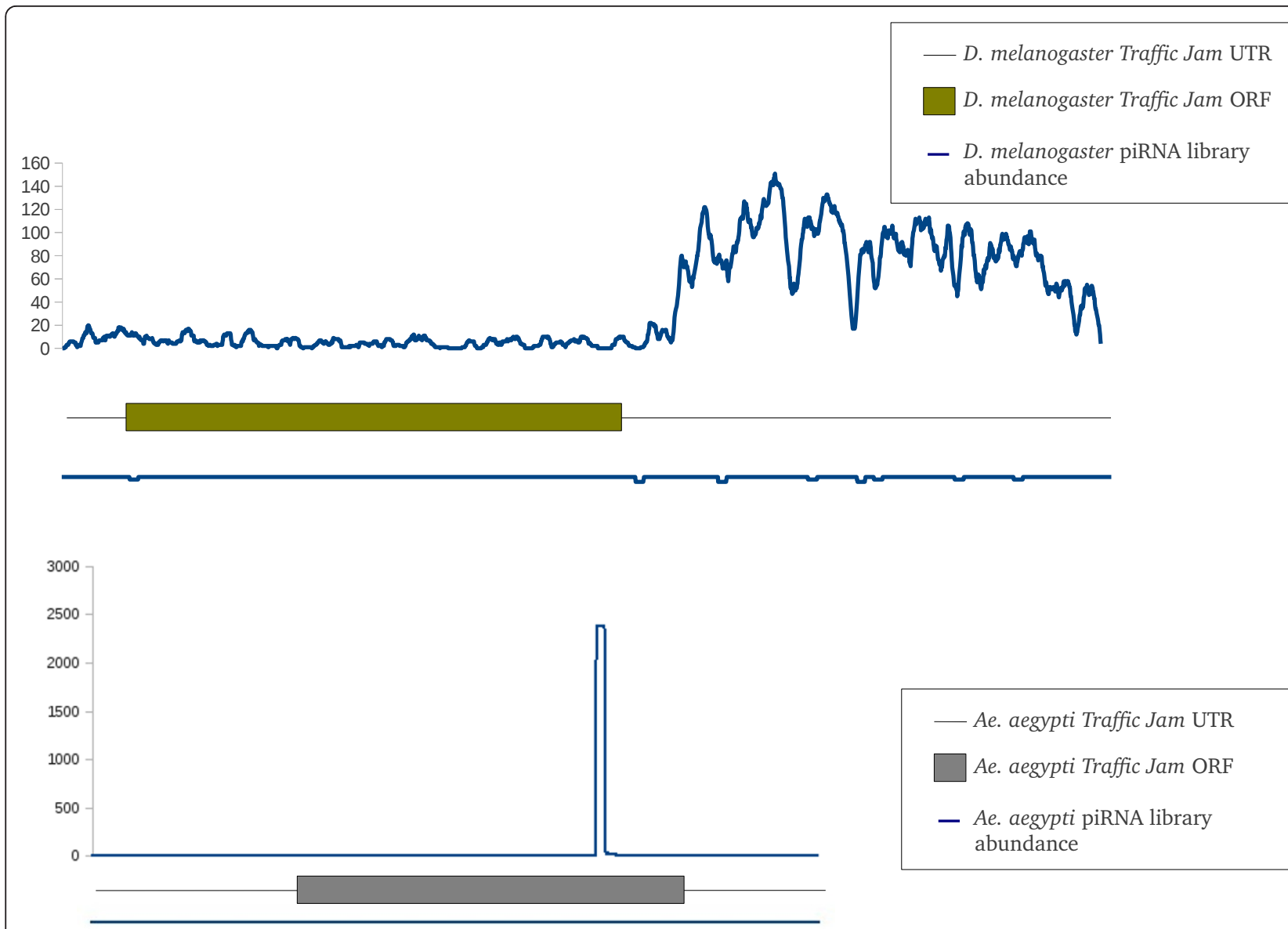

Figure 8 The abundance of $D$. melanogaster piRNA sequences mapping to the sense and antisense strands of the $D$. melanogaster t $j$ gene (top) and Ae. aegypti piRNA sequences (all libraries combined) mapping to the Ae. aegypti homolog of the tj gene (bottom) Abundance scale is shown on the left.

mapping to mael and found low numbers of them throughout the transcript, mapping mainly to the sense strand (Figure 9).

\section{Discussion}

High-throughput sequencing technology has greatly increased the opportunities to study the possible regulatory roles of piRNA and siRNA sequences. In arthropods most of the research has focused on the piRNA sequences of $D$. melanogaster (reviewed in $[13,58]$ ), where it has emerged that a major role of piRNAs is the regulation of transposons. However, D. melanogaster has a relatively small percentage of its genome composed of transposon derived sequences and has no described MITEs which are present in many of other insect genomes (including other drosophilids). We have conducted an analysis of piRNAs in the mosquito Ae aegypti, which is a vector of many arboviruses and contains a genome rich in transposon derived sequences (47\%) which includes a significant percentage of MITEs derived sequences (16\%) [16].

Sequencing of seven whole tissue libraries sampled only a portion of all available piRNA sequences in this mosquito. Based on sequence overlaps between these libraries we estimated that the total diversity of piRNA sequences in Ae. aegypti was within the same order of magnitude as the piRNA diversity of $D$. melanogaster. Given this similarity in overall piRNA diversity between Ae. aegypti and D. melanogaster it was surprising that only $19 \%$ of $A e$. aegypti piRNAs mapped to annotated transposon sequences, compared to $50 \%$ of piRNAs in our D. melanogaster library. Barring a large number of unrecognized transposons in Ae. aegypti, this suggests that the role of piRNAs in transposon regulation in Ae. aegypti follows the $D$. melanogaster model in some, but not all, respects.

The majority of the $19 \%$ of piRNAs that did map to transposons in Ae. aegypti mapped to the antisense transcription strand, a pattern that was previously observed 


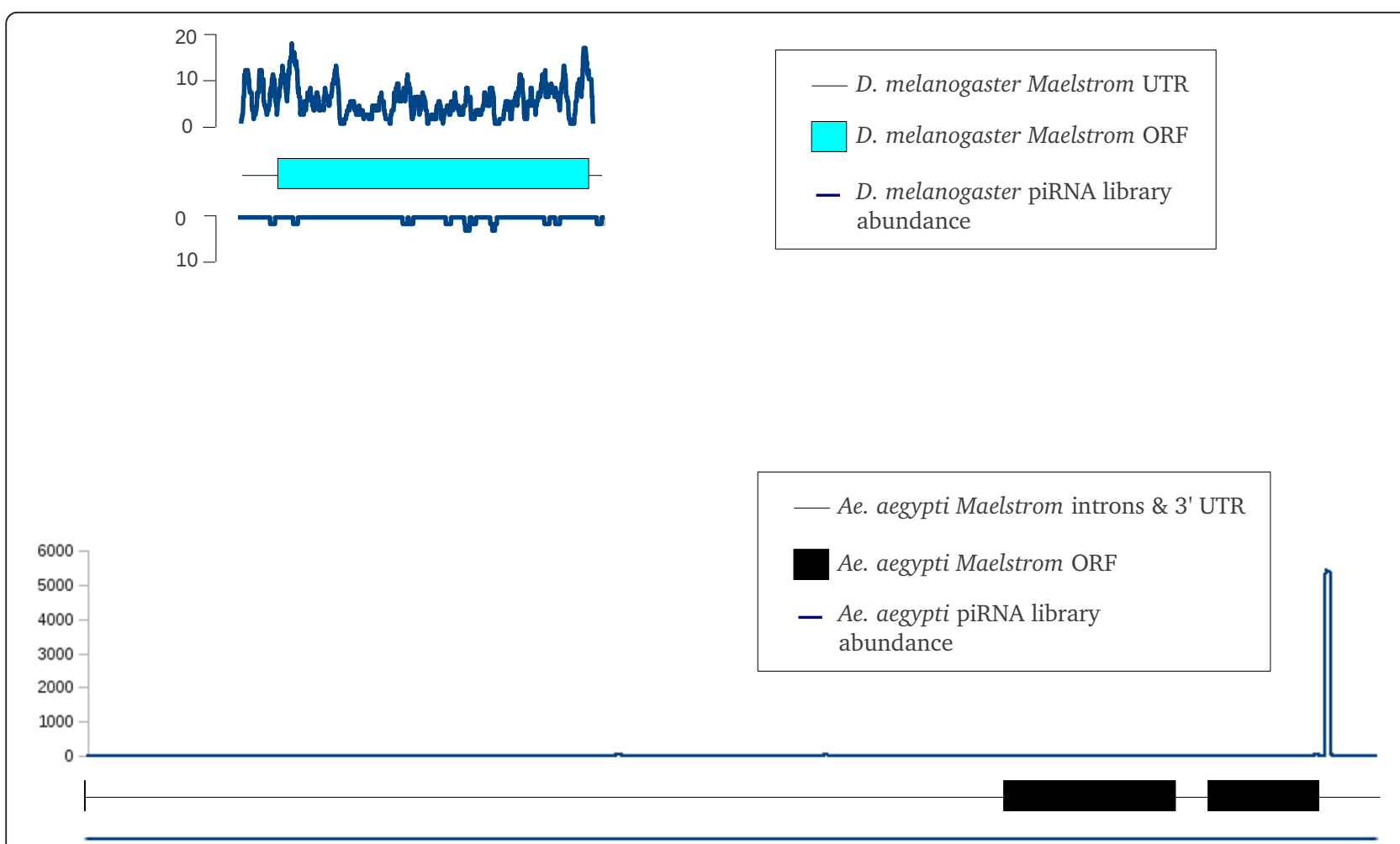

Figure 9 The abundance of $D$. melanogaster piRNA sequences mapping to sense and antisense strands of the $D$. melanogaster maelstrom gene (top) and Ae. aegypti piRNA sequences (all libraries combined) mapping to the Ae. aegypti homolog of the maelstrom gene (bottom). Abundance scale is shown on the left.

in D. melanogaster, and is consistent with having transposon transcripts sliced by PIWI proteins loaded with piRNAs that are antisense to the transposon transcript [31]. Ae aegypti piRNA sequences mapped to all transposon classes including MITEs (Table 2). MITEs may be transcribed but do not produce a functional protein product and so, in order to be mobilized require the presence of a corresponding full length and active transposase [67]. Ae. aegypti MITEs show little sequence similarity to full length transposons making it unlikely that many of the observed piRNAs mapping to MITEs are simply remnants of full length transposon inactivation systems. However, only $0.26 \%$ of Ae aegypti piRNAs mapped to MITEs despite them comprising $16 \%$ of the assembled genome. Conversely $4 \%$ of Ae. aegypti endo-siRNAs mapped to MITEs which is perhaps not surprising given that transcription along the length of a MITE would produce a foldback dsRNA sequence that would elicit the siRNA response. A link between transposons, miRNAs and gene regulation was first proposed from studies in humans and has been refined, based on analyses performed in plant genomes, to include both siRNAs and MITEs [68-71].

We were able to identify a number of piRNA clusters that generated a large percentage $(84 \%)$ of observed
piRNAs. A number of lines of evidence supported these genomic locations as having a similar role to $D$. melanogaster clusters. First, previously described D. melanogaster clusters were also recovered using our cluster discovery procedure applied to our $D$. melanogaster library (Table 3). Second, all Ae. aegypti libraries supported the same basic piRNA cluster locations (Table 4). Third, the top 30 Ae. aegypti clusters appeared to overlap with transcribed portions of the genome (Figure 5). Finally, these clusters shared some common features with previously described $D$. melanogaster piRNA clusters. These included: roughly similar ranges in individual cluster lengths; a mixture of clusters with piRNAs mapping almost exclusively to one strand and clusters with piRNAs mapping to both strands; and similarities in the relative orientation of piRNA, transposon, and transcription orientation for at lease some clusters. Some piRNA clusters in D. melanogaster and mouse are transcribed unidirectionally. This is the case for the D. melanogaster flamenco locus in which $99 \%$ of piRNAs map to the sense strand of transcription, while all the transposon sequences are oriented in the direction opposite to transcription [31]. Many of the top Ae. aegypti piRNA clusters also contained transposon sequences predominantly oriented in the same direction (Table 5). Unfortunately, 
overall cluster transcription direction could not be determined for all clusters. However, among the 30 piRNA clusters in Table 4 three overlapped with protein coding genes that were also identified as generating piRNAs (cluster 1, gene AAEL010454; cluster 3, genes AAEL007861 and AAEL007866; cluster 29, gene AAEL006159; Tables 4 and 7). If we assume that the clusters are transcribed in the same orientation as these genes we see a similar pattern in clusters 1 and 3 to the D. melanogaster flamenco locus: piRNAs are on the sense strand of transcription while transposon sequences are oriented in the opposite direction to transcription (in cluster 29 transposon were not predominantly oriented in any one direction). These observations further reinforce the similarity between Ae. aegypti and D. melanogaster piRNA clusters. However, while piRNA clusters in D. melanogaster and mouse generate sequences that predominantly map to transposons $[4,6]$ fewer than a quarter of potential piRNAs generated from Ae. aegypti clusters matched known transposons. Furthermore, while many previously described piRNA clusters contained a high density of transposon sequences we did not detect significantly higher levels of transposon sequences within Ae. aegypti clusters compared to random portions of the genome (Table 5). However Ae. aegypti piRNA clusters covered a greater portion of the assembled genome than D. melanogaster clusters and so may be more widespread. These results suggest that while Ae. aegypti and D. melanogaster share many features of their piRNA clusters, the role these clusters have in transposon inactivation may not be completely identical between these species. The nature of this difference has yet to be determined.

We examined the piRNA density maps to seven $A e$. aegypti transposons and found these to be similar in their patterns to equivalent density maps from $D$. melanogaster $[6,9,35]$. All but the non-LTR MosquI element contained at least on ping-pong amplification overlap suggesting that these could be silenced by this pathway. Notably these ping-pong signatures were present in representatives of the two LTR elements that together account for $58 \%$ of piRNAs. The most marked antisense strand bias was observed for the non-LTR JuanA element which has been proposed to be recently active in mosquitoes [52]

To better understand the role of the $81 \%$ of $A e$. aegypti piRNAs that did not map to transposons we examined possible associations between protein coding genes and piRNA sequences. In addition to their role in transposon transcript degradation, piRNA sequences have been demonstrated to silence protein coding genes in D. melanogaster $[60,72]$. The Supressor of Stellate $(\mathrm{Su}$ (ste)) and Traffic Jam ( $t j)$ genes contain piRNA sequences on their sense strand that can be used to degrade the transcripts of the Stellate (Ste) and Fasciclin 3 (Fas 3) gene transcripts respectively [60,72]. Ae. aegypti contained a number of genes with piRNAs mapping almost exclusively to their sense strand that are therefore unlikely to be involved in the regulation of the host gene transcript (Table 7). Instead, they may be used to regulate other genes, the identity of which cannot be deduced using the present data. However, it is interesting to note that just as the D. melanogaster $t j$ gene has been identified as a source of piRNAs, a possible ortholog of $t j$ in Ae aegypti may also be a source of piRNA sequences (AAEL007686, Table 7). Furthermore, a putative ortholog to the D. melanogaster Fas 3 gene has been identified in Ae. aegypti (AAEL003044, OrthoDB http://cegg.unige.ch/orthodb4) suggesting that the Ae. aegypti AAEL007686 gene has at least the potential to act in a similar way to the $D$. melanogaster $t j$ gene. One difference in the location of the sensestrand piRNAs arising from both Ae. aegypti and D. melanogaster is that for Ae. aegypti t $j$ they are located upstream from the translation termination codon rather than being located within the 3' UTR.

The location of piRNAs to the sense strand of the 3' UTR of the Ae. aegypti maelstrom (mael) gene suggests that these piRNAs may also be involved in the regulation of downstream genes, as had been proposed for $D$. melanogaster $t j[60]$ However as yet we are unable to identify these target genes. In D. melanogaster, mael is associated with both the nucleus and nuage of germ line cells [64]. Mutations in Mael, as well as in other components of the nuage, such as vasa and Krimper, have been shown to reduce the levels of piRNAs associated with the $H e T-A$, roo and $I$ transposons suggesting that these genes play a role in the suppression of transposition in the D. melanogaster female germ line [66]. Consistent with this is the role of Mael in mouse spermatogenesis in which its absence led to a 100 -fold increase in L1 expression and a 3-5 fold increase in the expression of the unrelated IAP element [73]. As was seen for both $A e$, aegypti and D. melanogaster t $j$, the piRNAs map to the sense strand of mael but differ from Ae. aegypti $t j$ in that they are located in the 3' UTR. We find it encouraging that two of the top 30 piRNA generating Ae. aegypti genes have previously implicated in the regulation of either piRNAs or transposons in D. melanogaster and so suggests that our own bioinformatics screening of these libraries is generating valid targets. Seven other protein coding genes also generated piRNAs only to the sense strand and all remain unannotated (AAEL011224, AAEL017228, AAEL005277, AAEL005213, AAEL009263, AAEL013013, AAEL011027)(Table 7). It remains to be determined if piRNAs from many of these genes arise from the exonic sequence since many current $A e$ a agypti gene 
annotations have not been manually curated and are based mostly on relatively poor EST datasets. Robine et al. (2009) noted that in mouse piRNA libraries many piRNA clusters that were once believed to be exonic could, upon reexamination, be reclassified as 3'UTR directed. This same phenomenon of high density of piRNAs on the sense strand of the 3' end of the ORF was also observed in the case of the putative maelstrom homolog gene described above.

Ae. aegypti is a vector of many RNA viruses, some of which cause severe disease in humans. Eight of the 30 top piRNA generating genes in Ae. aegypti are apparently of viral origin (Table 7). Three of these generate piRNAs only to the antisense strand (AAEL007866, AAEL017001, AAEL007844,) while another (AAEL009873) generates 99\% of it's piRNA to this strand. The remaining three (AAEL017355, AAEL000120, AAEL001772) generate piRNAs mapping to both strands, although for each it is the antisense strand that dominates. This may indicate that most of the piRNAs generated to viral-like genes function by, in association with the appropriate Piwi protein, slicing the viral gene transcripts. As such this mechanism is entirely different to that operating for $t$, mael and the seven other protein coding genes in which the piRNAs are generated exclusively by the sense strand. All of the remaining 12 protein coding genes that generate piRNAs have these mapping primarily to the antisense strand.

There has been some recent evidence implicating piRNAs in the recognition of arboviruses in Ae. aegypti and Aedes albopictus. In addition to siRNAs, small RNAs 24-30 nt in length to the sense strand of the dengue virus genome were recovered from infected Ae. aegypti and none showed a bias for uracil at the 5' end and little bias for adenine at the $10^{\text {th }}$ position although these authors stated that unpublished data revealed that small RNAs of the same size distribution generated to the sense strand of Sindbis virus did show a U1 bias [63]. Aedes albopictus C6/36 cells have been found to lack an siRNA response to infection by West Nile virus, Sindbis virus and La Crosse virus, but do generate small peaks of smaller RNAs 24-28 nt in size to Sindbis and La Crosse virus infections yet no such peak is generated by infection with the dengue virus $[62,74]$. Interestingly small RNAs within this size range generated to the inadvertent infection of C6/36 cells by Cell Fusing Agent virus showed a strong preference for adenine at the $10^{\text {th }}$ position which is consistent with them being piRNAs that interact with, in D. melanogaster, the AGO3 protein [74]. Taken together these data from two difference Aedes species indicate that a piRNA response to arboviral infection may be generated and, if so, implicate this pathway in an anti-viral response. Taken in this context, the piRNAs generated by the viral-like sequences identified here may be further evidence of the role that this small RNA pathway may play in anti-viral defense in this mosquito. Ae aegypti may thus provide important and novel information concerning how this small RNA pathway interacts with both transposons and viruses, both of which are abundant in this insect, especially in comparison with $D$. melanogaster.

A large portion of transposon-matching Ae. aegypti piRNAs mapped inside genic sequences for reasons that remain unclear (Figure 1A). Some protein coding genes are likely origins of piRNAs (Table 7), but it appears unlikely that these would be sufficient in number to account for this observation. A possible, but as yet untested, explanation might be that these regions contain a high level of active transposons. Genic regions of the genome are more likely to be transcribed, which may increase the chances that an inserted transposon will actively transpose. This in turn could produce a higher response of piRNA silencing mechanism to these transposons.

As a vector of human disease pathogens there is interest in developing highly robust genetic tools for $A e$. aegypti. While germ line transformation is possible (albeit a low rate compared to D. melanogaster) efforts to remobilize transposons in Ae. aegypti have occurred at very low rate suggesting the presence of a transposon silencing mechanism $[21,23,41,75]$. We examined the piRNA content of mosquito lines that had been transformed with transposon sequences and found preliminary evidence that piRNA sequences mapping exclusively to the transformed sequences had been produced. Since these piRNAs did not match the current Ae. aegypti assembly their presence in the transformed lines was likely explained in one of two ways: 1 ) they had been maternally inherited and perhaps amplified via the pingpong cycle, or 2) new piRNAs were being generated from introduced sequence. In either case these data are suggestive that a component of the piRNA pathway was activated by the insertion of foreign DNA into the genome although we have no information as to how rapid this response would have occurred. The full kinetics of the piRNA response to transgenic sequences need to be explored in association with genome-wide transcriptional analyses which should shed light on the relationship between transgenesis, the small RNA response and viral infection in this mosquito

\section{Conclusions}

We analyzed piRNA and endo-siRNA sequences from Ae. aegypti, a mosquito that is a significant vector of human pathogens and has a large genome size with a correspondingly high transposon content. Unlike $D$. melanogaster, Ae. aegypti contains MITEs and we found 
higher levels of siRNAs targeted to these than piRNAs. The terminal inverted repeats of MITEs most likely enables foldback RNAs to be formed from unidirectional transcripts, leading to the induction of the siRNA pathway, which is associated more with anti-viral defense. Despite having an abundance of transposons, the majority of piRNAs in Ae. aegypti were targeted to non-transposon sequences, many of which were protein-coding genes. As such the piRNA profile of this mosquito is more similar to that of mice than D. melanogaster in which the majority of piRNA sequences map to transposons. The majority of piRNAs in this mosquito were 28 nt in length and so longer than those seen in D. melanogaster but contained the U1 or A10 sequence bias seen in other organisms in which piRNAs have been sequenced. Two genes targeted by piRNAs in $A e$. aegypti have been implicated in piRNA biogenesis or function while the function of the majority of them remain unknown. Several others were of viral origin suggesting that the piRNA response may extend into anti-viral defense in this insect. piRNAs were also generated to introduced transposons. The diversity of endogenous transposons present in this mosquito, together with the corresponding diversity and number of piRNAs and siRNAs mapping to them suggests that these small RNA pathways may of some importance in maintaining the integrity of its genome in the presence of numerous transposons and viruses.

\section{Methods}

\section{Purification of Small RNAs from Ae. aegypti and D.} melanogaster/Library Construction

Total RNA was extracted from approximately 200 mosquitoes using Trizol reagent (Invitrogen). 10-20 $\mu \mathrm{g}$ of the total RNA was run on a $15 \%$ polyacrylamide/7M urea/ TBE gel using a Hoeffer SE420 electrophoresis apparatus (Hoeffer). Gel bands corresponding to approximately 16 to 35 bases were excised. The Illumina small RNA sample prep kit (Illumina) was used for all steps of library construction. Gel bands were broken up by centrifugation through small holes in $0.5 \mathrm{ml}$ microfuge tubes and RNA was eluted with $0.3 \mathrm{M} \mathrm{NaCl}$. Following gel removal with Spin-X filters and precipitation with glycogen and ethanol, samples were resuspended in water. Small RNAs were ligated to the SRA 5' adapter overnight before size selection on a $15 \%$ polyacrylamide/ $7 \mathrm{M}$ urea/TBE gel. Gel bands corresponding to approximately 40-60 bases were excised and purified as above. The samples were next ligated overnight to the 3' adapter before purification on a $10 \%$ polyacrylamide/7ㅆ urea/TBE gel. Gel bands corresponding to 70-90 bases were excised and purified. Reverse transcription was performed using Superscript III (Invitrogen) before library amplification with Phusion DNA polymerase.
Amplification was as follows: $98^{\circ} \mathrm{C}$ for 30 seconds, followed by 15 cycles of: $98^{\circ} \mathrm{C} 10$ seconds, $60^{\circ} \mathrm{C}$ for $30 \mathrm{sec}-$ onds, and $72^{\circ} \mathrm{C}$ for 15 seconds, with a final step of 10 minutes at $72^{\circ} \mathrm{C}$. The final library was purified by size selection of gel bands corresponding to $85-110 \mathrm{bp}$ on a $6 \%$ polyacrylamide/TBE gel. Library quality was assessed by ligation into the pJET1.2 vector (CloneJet kit, Fermentas) followed by standard DNA sequencing. Final library sequencing was performed by the staff of the UCR Institute for Integrative Genome Biology on the Illumina GAx2 sequencer.

\section{Processing libraries}

Sequences were bioinformatically stripped of adapters using $\mathrm{R}$ scripts. Following this ribosomal sequences were removed by mapping each library to a database containing all known ribosomal RNAs (rRNA, tRNA, snRNA, etc.) derived from Genbank records (http:// www.ncbi.nlm.nih.gov/genbank/) for the appropriate genome and removing any sequences with significant matches. A similar process was used to remove miRNAs using the sequences deposited in mirBase (http://www. mirbase.org/) to identify Ae. aegypti and D. melanogaster miRNAs. Finally, sequences were mapped (see below) either to the Ae. aegypti assembly available at VectorBase (http://www.vectorbase.org) or to the BDGP Release 5 D. melanogaster assembly (http://www.fruitfly. org/). All analyses were limited to sequences that mapped to the reference genome with the exception of the analysis of piRNAs mapping to transformed sequences, where all piRNAs were used.

\section{Mapping sequences to genomes and other databases}

Mapping was mostly performed using the program Bowtie [76]. Mapping to the genome was performed using a seed length of $30 \mathrm{bp}$ and allowing up to 2 mismatches within the seed. Mapping to other databases did not use a seed, but instead required a match along the entire length of the sequence with up to $2 \mathrm{bp}$ mismatches. The only mapping not performed using Bowtie, was matching the sequenced libraries to ribosomal databases (see above) which was performed using the BLAT program with the "-fastMap" option [77].

\section{Estimating piRNA abundance}

We based our estimates of the size of the piRNA sequence pool in Ae. aegypti and D. melanogaster on the observed number of piRNAs in each library (i.e. sequences $24 \mathrm{nt}$ long or larger) and on the amount of overlap between libraries. These were used with the formula described previously [45]. However, based on simulation experiments we designed to verify this method in-silico it appeared that including highly duplicated sequences into the calculation could have a large 
negative impact on the estimates. We minimized this effect by excluding from the analysis any sequence that was duplicated in any one library. We estimated the size of Ae. aegypti piRNA pool using every possible pair of Ae. aegypti libraries that were not replicates (19 estimates) and averaged these for a final estimate of the piRNA pool size. For D. melanogaster we used eight published libraries [46] deposited at the National Center for Bioinformatic Infomation GEO database under record GSE30955. Only sequences that were $24 \mathrm{nt}$ long or larger and that mapped to D. melanogaster genome assembly were used from these eight libraries to minimize the chances that non-piRNA sequences were included. Estimates were performed on all possible pairs of the eight libraries (28 estimates).

\section{Periodate oxidation and $\beta$-elimination of small RNAs}

For analysis of the chemical structure of the 3' ends of small RNAs from Ae. aegypti, total RNA was purified using Trizol reagent (Invitrogen) from 2 day postblood-fed females. RNAs of approximately 28-32 nt were purified from $10 \mu \mathrm{g}$ total RNA on a $15 \%$ polyacrylamide gel containing 7.5 M urea. RNA ladder was obtained from Illumina, and the control 23-mer synthetic RNA was the kind gift of Dr. Shou-wei Ding (University of California, Riverside). Following removal of 5'-phophates with FastAP (Fermentas), RNAs were labeled with ${ }^{32} \mathrm{P}-\Upsilon$-ATP and T4 polynucleotide kinase (Fermentas). The method for the $\beta$-elimination was according to published protocols [32]. Signals were visualized with BioMax film (Kodak).

\section{Additional material}

Additional file 1: Figure S1. Size distribution of Ae. aegypti small RNA abundance in each of the seven Ae. aegypti libraries and the single $D$. melanogaster library. The number of small RNAs mapping to Ae. aegypti genes, transposons, both, or neither, are shown as different colors for each size class (the legend is shown on the right).

Additional file 2: Table S1. Number of piRNAs from Ae. aegypti libraries mapping to Transposable Element (TE) family consensus sequences and percentage occupancy of the genome by TE families.

Additional file 3: Figure S1. Small RNA ( $>=24 \mathrm{nt}$.) density plots for representative Ae. aegypti full length transposable elements. Small RNA density for all sequenced Ae. aegypti libraries mapping to the sense strand of the transposable element is show in red, mapping to the antisense strand shown in blue. Position and density of possible U1A10 overlap pairs is shown in light blue. Position of the ORF(s) is shown at the bottom of each figure.

Additional file 4: Figure S1. piRNA density, mRNA-seq transcript coverage and assembly of the Ae. aegypti genomic region surrounding a putative Ae. aegypti homolog of the traffic jam gene. Genomic region supercontig identity and boundaries are shown below the piRNA density graph. mRNA-seq data were derived from an Ae. aegypti ovary tissue library. mRNA-seq transcript assembly, shown in blue, was performed using the CUFFLINKS program (Trapnell et al. 2010). Location on the genomic region of the mRNA transcript assembly and gene annotation, as reported in VectorBase, are show at the bottom.
Additional file 5: Figure S1. piRNA density, mRNA-seq transcript coverage and assembly of the Ae. aegypti genomic region surrounding a putative Ae. aegypti homolog of the MAELSTROM gene. Genomic region supercontig identity and boundaries are shown below the piRNA density graph. mRNA-seq data were derived from an Ae. aegypti ovary tissue library. mRNA-seq transcript assembly, shown in blue, was performed using the CUFFLINKS program (Trapnell et al. 2010). Location on the genomic region of the mRNA transcript assembly and gene annotations, as reported in VectorBase, are show at the bottom. Reference cited in Additional file 5, Figure S1. 1. Trapnell et al., "Transcript assembly and quantification by RNA-Seq reveals unannotated transcripts and isoform switching during cell differentiation," Nature Biotechnology 28, no. 5 (2010): 511-515.

\section{Acknowledgements \& Funding}

This research was supported by award 1R56A1088852-01A1 to PWA under the American Recovery and Reinvestment Act of 2009. Members of the Atkinson laboratory are thanked for their helpful discussions. We thank David A. O'Brochta of the University of Maryland for strain pMos3DBhspPBac.

\section{Author details}

${ }^{1}$ Center for Disease Vector Research, Institute for Integrative Genome Biology, and Department of Entomology, University of California, Riverside, CA 92521, USA. ${ }^{2}$ Department of Molecular Biology \& Genetics and Howard Hughes Medical Institute, Johns Hopkins School of Medicine, Baltimore, MD 20742,USA.

\section{Authors' contributions}

PA carried out bioinformatics analyses, RHH performed molecular genetic studies and library constructions, JAW participated in molecular genetic analyses, library constructions, and bioinformatic analysis, NLC participated in experimental design and edited the manuscript, PWA conceived of the study, developed the experimental design and wrote the manuscript with editorial assistance from all co-authors who approved the final manuscript.

Received: 2 September 2011 Accepted: 15 December 2011

Published: 15 December 2011

\section{References}

1. Senti K-A, Brennecke J: The piRNA pathway: a fly's perspective on the guardian of the genome. Trends Genet 2010, 26(12):499-509.

2. Saito K, Siomi MC: Small RNA-mediated quiescence of transposable elements in animals. Developmental Cell 2010, 19:687-697.

3. Siomi MC, Miyoshi T, Siomi H: piRNA-mediated silencing in Drosophila germlines. Semin Cel Dev Biol 2010, 21(7):754-759.

4. Aravin AA, Sachidanandam R, Bourc'his D, Schaefer C, Pezic D, Fejes-Toth K, Bestor T, Hannon GJ: A piRNA pathway primed by individual transposons in linked to de novo methylation in mice. Mol Cell 2008, 31:785-799.

5. Houwing S, Kamminga LM, Berezikov E, Cronembold D, Girard A, van den Elst H, Filippov DV, Blaser H, Raz E, Moens CB, et al: A role for Piwi and piRNAs in germ cell maintenance and transposon silencing in zebrafish. Cell 2007, 129:69-82.

6. Brennecke J, Aravin AA, Stark A, Dus M, Kellis M, Sachidanandam R, Hannon GJ: Discrete small RNA-generating loci as master regulators of transposon activity in Drosophila. Cell 2007, 128(6):1089-1103.

7. Vagin W, Sigova A, C L, Gvozdev V, Zamore PD: A distinct small RNA pathway silences selfish genetic elements in the germline. Science 2006, 313:320-324.

8. Siomi MC, Sato. K, Pezic D, Aravin AA: PIWl-interacting small RNAs: the vanguard of genome defence. Nature Reviews: Mol Cell Biol 2011, 12:246-258.

9. Malone CD, Brennecke J, Dus M, Stark A, McCombie WR, Sachidanandam R, Hannnon GJ: Specialized piRNA pathways act in germline and somatic tissues of the Drosophila ovary. Cell 2009, 137:522-535.

10. Chung W-J, Okamura K, Martin R, Lai EC: Endogenous RNA interference provdes a somatic defense against Drosophila transposons. Current Biology 2008, 18:795-802. 
11. Robine N, Lau NC, Balla S, Jin Z, Okamura K, Kuramochi-Miyagawa S, Blower MD, Lai EC: A broadly conserved primary pathway generates 3'UTR-direceted primary piRNAs. Current Biology 2009, 19:2066-2076.

12. Lau NC, Robine N, Martin R, Chung W-J, Niki Y, Berezikov E, Lai EC: Abundant primary piRNAs, endo-siRNAs, and microRNAs in a Drosophila ovary cell line. Genome Research 2009, 19(10):1776-1785.

13. Khurana JS, Theurkauf W: piRNAs, transposon silencing, and Drosophila germline development. J Cell Biol 2010, 191(5):905-913.

14. Kaminker JS, Bergman CM, Kronmiller B, Carlson J, Svirskas R, Patel S, Frise E, Wheeler DA, Lewis SE, Rubin GM, et al: The transposable elements of the Drosophila melanogaster euchromatin: a genomics perspective. Genome Biology 2002, 3:0084.0081-0084.0020.

15. Smith CD, Shu S, Mungall CJ, Karpen GH: The release 5.1 annotation of Drosophila melanogaster heterochromatin. Science 2007, 316:1586-1591.

16. Nene V, Wortman JR, Lawson D, Haas B: Genome sequence of Aedes aegypti, a major arbovirus vector. Science 2007, 316(5832):1718-1723.

17. Kokoza V, Ahmed A, Wimmer EA, Raikhel AS: Efficient transformation of the yellow fever mosquito Aedes aegypti using the piggyBac transposable element vector pBac[3xP3-EGFPafm]. Insect Biochem Mol Biol 2001, 31:1137-1143.

18. Nimmo DD, Alphey L, Meredith JM, P E: High efficiency site-specific engineering of the mosquito genome. Insect Mol Biol 2006, 15:129-136.

19. Attardo $G M$, Higgs $S$, Klingler KA, Vanlandingham DL, Raikhel AS: RNA interference-mediated knockdown of a GATA factor reveals a link to anautogeny in the mosquito Aedes aegypti. Proc Natl Acad Sci USA 2003, 100(23):13374-13379.

20. Clemons A, Haugen M, Severson D, Duman-Scheel M: Functional analysis of gene in Aedes aegypti embryos. Cold Spring Harb Protoc 2010.

21. Smith RC, Atkinson PW: Mobility properties of the Hermes transposable element in transgenic lines of Aedes aegypti. Genetica 2010, 139(1):7-22.

22. O'Brochta DA, Sethuramuran $N$, Wilson $R$, Hice $R H$, Pinkerton $A C$, Levesque CS, Bideshi DK, Jasinskiene N, Coates CJ, James AA, et al: Gene vector and transposable element behavior in mosquitoes. Journal of Experimental Biology 2003, 3823-3834.

23. Wilson R, Orsetti J, Klocko AK, Aluvihare C, Peckham E, Atkinson PW Lehane MJ, O'Brochta DA: Post-integration behavior of a Mos1 mariner gene vector in Aedes aegypti. Insect Biochem Mol Biol 2003, 33:853-863.

24. Sethuraman N, Fraser MJ Jr, Eggleston P, O'Brochta DA: Post-integration stability of piggyBac in Aedes aegypti. Insect Biochem Mol Biol 2007 37(9):941-951.

25. Trauner J, Schinko J, Lorenzen MD, Shippy TD, Wimmer EA, Beeman RW Klingler M, Bucher G, Brown SJ: Large-scale insertional mutagenesis of a coleopteran stored grain pest, the red flour beetle Tribolium castaneum, identifies embryonic lethal mutations and enhancer traps. BMC Biology 2009, 7:73

26. Thibault ST, Singer MA, Miyazaki WY, Milash B, Dompe NA, Singh CM, Buchholz R, Demsky M, Fawcett R, Francis-Lang HL, et al: A complementary transposon tool kit for Drosophila melanogaster using $P$ and piggyBac. Nat Genet 2004, 36(3):283-287.

27. Ding S, Wu X, Li G, Han M, Zhuang Y, Xu T: Efficient transposition of the piggyBac $(P B)$ transposon in mammalian cells and mice. Cell 2005, 122:473-483.

28. O'Donnell KA, Boeke JD: Mighty Piwia defend the genome against genome intruders. Cell 2007, 129:37-44.

29. Vagin W, Klenov MS, Kalmykova Al, Stolyarenko AD, Kotelnikov RN, Gvozdev VA: The RNA interference proteins and vasa locus are involved in the silencing of retrotransposons in the female germline of Drosophila melanogaster. RNA Biol 2004, 1(1):54-58.

30. Cox DN, Chao A, Lin H: Piwi encodes a nucleoplasmic factor whose activity modulates the number and divsion rate of germ-line stem cells. Development 2000, 127:503-514.

31. Brennecke JB, Aravin AA, Stark A, Dus M, Kellis M, Sachidanandam R, Hannon GL: Discrete small RNA-generating loci as master regulators of transposon activity in Drosophila. Cell 2007, 128:1089-1103.

32. Gunawardane LS, Saito K, Nishida KM, Miyoshi K, Kawamura Y, Nagami T, Siomi $H$, Siomi MC: A slicer-mediated mechanism for repeat-associated siRNA $5^{\prime}$ end formation in Drosophila. Science 2007, 315(5818):1587-1590.

33. Saito K, Sakaguchi $Y$, Suzuki T, Suzunki T, Siomi H, Siomi MC: Pimet, the Drosophila homolog of HEN1, mediates 2'-O-methylation of Piwiinteracting RNAs at their 3' ends. Genes \& Development 2007. 21:1603-1608.
34. Horwich MD, Li C, Matranga C, Vagin V, Farley G, Wang P, Zamore PD: The Drosophila RNA methyltransferase, DmHen1, modifies germline piRNAs and single-stranded siRNAs in RISC. Current Biology 2007, 17:1265-1272.

35. Brennecke J, Malone CD, Aravin AA, Sachidanandam R, Stark A, Hannon GJ: An epigenetic role for maternally inherited piRNAs in transposon silencing. Science 2008, 322:1387-1392.

36. Jensen PA, Stuart JR, Goodpaster MP, Goodman JW, Simmons MJ: Cytoptype regulation of $P$ transposable elements in Drosophila melanogaster: repressor polypeptides or piRNAs? Genetics 2008, 179:1785-1793.

37. Campbell CL, Black WCBI, Hess AM, Foy BD: Comparative genomics of small regulatory pathway components in vector mosquitoes. BMC Genomics 2008, 9:425.

38. Carthew RW, Sontheimer EJ: Origins and mechanisms of miRNAs and siRNAs. Cell 2009, 136:632-655.

39. Smith RC, Walter MF, Hice RH, O'Brochta DA, Atkinson PW: Testis-specific expression of the $B 2$ tubulin promoter of Aedes aegypti and its application as a genetic sex-separation marker. Insect Mol Biol 2007, 16:61-71.

40. O'Brochta DA, Stosic CD, Pilitt K, Subramanian RA, Hice R, Atkinson PW: Transpositionally active episomal hAT elements. BMC Mol Biol 2009, 14(10):108.

41. Sethuraman N, Fraser MJJ, Eggleston P, O'Brochta DA: Post-integration stability of piggyBac in Aedes aegypti. Insect Biochem Mol Biol 2007, 37(9):941-951.

42. Malone CD, Hannon GJ: Small RNAs as guardians of the genome. Cell 2009, 136:656-668.

43. Kawaoka S, Hayashi N, Katsuma S, Kishino H, Kohara Y, Mita K, Shimada T: Bombyx small RNAs: Genomic defense system against transposon in the silkworm, Bombyx mori. Insect Biochem Molec Biol 2008.

44. Saito K, Nishida KM, Mori T, Kawamura Y, Miyoshi K, Nagami T, Siomi H, Siomi MC: Specific association of Piwi with rasiRNAs derived from retrotransposon and heterochromatic regions in the Drosophila genome. Genes Dev 2006, 20:2214-2222.

45. Betel D, Sheridan R, Marks DS, Sander C: Computational analysis of mouse piRNA sequence and biogenesis. PLoS Comput Biol 2007, 3(11):e222

46. Handler D, Olivieri D, Novatchkova M, Gruber FS, Meixner K, Mechtler K, Stark A, Sachidanaandam R, Brennecke J: A systematic analysis of Drosophila TUDOR domain-containing proteins identifies Vreteno and the Tdrd12 family as essential primary piRNA pathway factors. EMBO J 2011, 30:3977-3993.

47. Rozhkov NV, Aravin AA, Zelentsova ES, Schostak NG, Sachidanandam R, McCombie WR, Hannon GJ, Evgen'ev MB: Small RNA-based silencing strategies for transposons in the process of invading Drosophila species. RNA 2010, 16:1634-1645.

48. Gibbons JG, Janson EM, Hittinger $C T$, Johnston $M$, Abbot $P$, Rokas $A$ : Benchmarking next-generation transcriptome sequencing for functional and evoltionary genomics. Mol Biol Evol 2009, 26(12):2731-2744.

49. Jurka J: Repbase update: a database and an electronic journal of repetitive elements. Trends Genet 2000, 16(9):418-420.

50. Arensburger $P$, Hice RH, Zhou L, Smith RC, Tom AC, Wright JA, J. K, O'Brochta DA, Craig NL, Atkinson PW: Phylogenetic and functional characterization of the $h A T$ transposon superfamily. Genetics 2011 188(1):45-57.

51. Coy MR, Tu Z: Genomic and evolutionary analyses of Tango transposons in Aedes aegypti, Anopheles gambiae and other mosquito species. Insect Mol Biol 2007, 16(4):411-421.

52. Biedler JK, Tu Z: The Juan non-LTR retrotransposon in mosquitoes: genomic impact, vertical transmission and indications of recent and widespread activity. BMC Evolutionary Biology 2007, 7:112.

53. Tu Z, Isoe J, Guzova JA: Strutural, genomic, and phylogenetic analyssis of Lian, a novel family of non-LTR retrotransposons in the yellow fever mosquito, Aedes aegypti. Mol Biol Evol 1998, 15(7).

54. Tu Z, Hill JJ: Mosqul, a novel family of mosquito retrotransposons distantly related to the Drosophila I factors, may consist of elements of more than one origin. Mol Biol Evol 1999, 16(12):1675-1686.

55. Czech B, Malone CD, Zhou R, Stark A, Schlingheyde C, Dus M, Perrrimon N, Kellis M, Wohlshlegel JA, Sachidanaandam R, et al: An endogenous small interfering RNA pathway in Drosophila. Nature 2008, 453:798-804. 
56. Ghildiyal M, Seitz H, Horwich MD, Li C, Du T, Lee S, Xu J, Kittler ELW, Zapp ML, Weng Z, et al: Endogenous siRNAs derived from transposons and mRNAs in Drosophila somatic cells. Science 2008, 320:1077-1081.

57. Kawamura Y, Saito K, Kin T, Ono Y, Asai K, Sunohara T, Okada TN, Siomi MC, Siomi H: Drosophila endogenous small RNAs bind to Argonaute 2 in somatic cells. Nature 2008, 453:793-798.

58. Siomi MC, Sato K, Pezic D, Aravin AA: PIWI-interacting small RNAs: the vanguard of genome defence. Nat Rev Mol Cell Biol 2011, 12(4):246-258.

59. Nishida KM, Saito K, Mori T, Kawamura Y, Nagami-Okada T, Inagaki $\mathrm{S}$, Siomi H, Siomi MC: Gene silencing mechanisms mediated by AuberginepiRNA complexes in Drosophila male gonad. RNA 2007, 13:1911-1922.

60. Saito K, Inagaki S, Mituyama T, Kawamura Y, Ono Y, Sakota E, Kotani H, Asai K, Siomi H, Siomi MC: A regulatory circuit for piwi by the large Maf gene traffic jam in Drosophila. Nature 2009, 461:1296-1299.

61. Myles KM, Wiley MR, Morazzani EM, Adelman ZN: Alphavirus-derived small RNAs modulate pathogenesis in disease vector mosquitoes. Proc Natl Acad, Sci USA 2008, 105(50):19938-19943.

62. Brackney DE, Scott JC, Sagawa F, Woodward JE, Miller NA, Schilkey FD, Mudge J, Wilusz J, Olson KE, Blair CD, et al: C6/36 Aedes albopictus cells have a dysfunctional antiviral RNA interference response. PLOS Neglected Tropical Diseases 2010, 4(10):e856.

63. Hess AM, Prasad AN, Ptitsyn A, Ebel GD, Olson KE, Barbacioru C, Monighetti C, Campbell CL: Small RNA profiling of dengue virusmosquito interactions implicates the PIWI RNA pathway in anti-viral defense. BMC Microbiology 2011, 11:45.

64. Findley SD, Tamanaha M, Clegg NJ, Ruohola-Baker H: Maelstrom, a Drosophila spindle-class gene, encodes a protein that colocalizes with Vasa and RDE1/AGO1 homolog, Aubergine, in nuage. Development 2003, 130:859-871

65. Soper SFC, van der Heijden GW, Hardiman TC, Goodheart M, Martin SL, de Boer $\mathrm{P}$, Bortvin A: Mouse maelstrom, a component of nuage, is essential for spermatogenesis and transposon repression in meosis. Developmental Cell 2008, 15:285-297.

66. Lim AK, Kai T: Unique germ-line organelle, nuage functions to repress selfish genetic elements in Drosophila melanogaster. Proc Natl Acad, Sci USA 2007, 104(16):6714-6719.

67. Feschotte $C$, Zhang $X$, Wessler S: Miniature inverted-repeat transposable elements (MITEs) and their relationship with established DNA transposons. In Mobile DNA II. Edited by: Craig NL, Craigie R, Gellert M, Lambowitz AM. Washington, DC: American Society for Microbiology Press; 2002:1147-1158

68. Kuang H, Padmanabhan C, Li F, Kamei A, Bhaskar PB, Ouyang S, Jiang J, Buell CR, Baker B: Identification of minature inverted-repeat transposable elements (MITEs) and biogenesis of their siRNAs in the Solanaceae: New functional impllications for MITEs. Genome Research 2009, 19:42-56.

69. Piriyapongsa J, Marino-Ramirez L, Jordan IK: Origin and evolution of human microRNAs from transposable elements. Genetics 2007, 176:1323-1337.

70. Piriyapongsa J, Jordan IK: Dual coding of siRNAs and miRNAs by plant transposable elements. RNA 2008, 14:814-821.

71. Liu J, He Y, Amasino R, Chen $X$ : siRNAs trageting an intronic transposin in the regulation of natural flowering behavior in Arabidopsis. Genes \& Development 2004, 18:2873-2878.

72. Aravin AA, Naumova NM, Tulin AV, Vagin W, Rozovsky YM, Gvozdev VA: Double-stranded RNA-mediated silencing of genomic tandem repeats and transposable elements in the D. melanogaster germline. Curr Biol 2001, 11(13):1017-1027.

73. Soper SFC, van der Heijden GW, Hardiman TC, Goodheart M, Martin SL, de Boer P, Bortvin A: Mouse Maelstrom, a component of nuage is essential for spermatogenesis and transposon repression in meiosis. Developmental Cell 2008, 15:285-297.

74. Scott JC, Brackney DE, Campbell CL, Bondu-Hawkins V, Hjelle B, Ebel GD, Olson KE, Blair CD: Comparison of dengue virus type 2-specific small RNAs from RNA interference-competent and -incompetent mosquito cells. PIOS NTM 2010, 4:(10)

75. O'Brochta DA, Sethuraman N, Wilson R, Hice RH, Pinkerton AC, Levesque CS, Bideshi dK, Jasinskiene J, Coates CJ, James AA, et al: Gene vector and transposable element behavior in mosquitoes. J Experimental Biol 2003, 206:3823-3834
76. Langmead B, Trapnell C, Pop M, Salzber SL: Ultrafast and memory-efficient alignment of short DNA sequences to the human genome. Genome Biol 2009, 10(3):R25.

77. Kent WJ: BLAT - the BLAST - like alignment tool. Genome Res 2002, 12:656-664.

78. Crooks GE, Hon G, Chandonia JM, Brenner SE: WebLogo: a sequence logo generator. Genome Res 2004, 14(6):1188-1190.

\section{doi:10.1186/1471-2164-12-606}

Cite this article as: Arensburger et al:: The mosquito Aedes aegypti has a large genome size and high transposable element load but contains a low proportion of transposon-specific piRNAs. BMC Genomics 2011 $12: 606$

\section{Submit your next manuscript to BioMed Central and take full advantage of:}

- Convenient online submission

- Thorough peer review

- No space constraints or color figure charges

- Immediate publication on acceptance

- Inclusion in PubMed, CAS, Scopus and Google Scholar

- Research which is freely available for redistribution

Submit your manuscript at www.biomedcentral.com/submit
Biomed Central 